\title{
Molecular Mobility of Amorphous N-Acetyl- $\alpha$-Methylbenzylamine and Debye Relaxation evidenced by Dielectric Relaxation Spectroscopy and Molecular Dynamics Simulations
}

\author{
Bienvenu Atawa †§, Natália T. Correiaキ, Nicolas Couvrat §, Frédéric Affouard‡*, \\ Gérard Coquerel §, Eric Dargent †, Allisson Saiter † \\ † Normandie Univ, UNIROUEN, INSA Rouen, CNRS, GPM, 76000 Rouen, France \\ $\S$ Normandie Univ, UNIROUEN, SMS, 76000 Rouen, France \\ f Univ Lille, CNRS, UMR 8207, UMET, Unité Matériaux et Transformations, F59000 \\ Villeneuve d'Ascq, France
}

*corresponding author

Email: frederic.affouard@univ-lille.fr

\begin{abstract}
The present work focusses on the molecular mobility characterization of amorphous $\mathrm{N}$-acetyl- $\alpha$-methylbenzylamine (Nac-MBA) by Broadband Dielectric Relaxation Spectroscopy (DRS) coupled to Fast Scanning Calorimetry (FSC) and Molecular Dynamics (MD) Simulations covering over 12 decades in the frequency range. This study reveals another example of a secondary amide that shows a very intense Debye-like contribution (almost $90 \%$ of the global dielectric intensity) in addition to the structural $\alpha$-relaxation and secondary Johari-Goldstein $\beta$-relaxation). The $D$ - and $\alpha$-relaxations are separated by about one decade (in frequency) and their relaxation times follow a near parallel temperature evolution (VogelFulcher-Tammann-Hesse). The micro-structure of Nac-MBA has been investigated from MD simulations. It is shown that intense Debye-like process emanates from the formation of linear intermolecular $\mathrm{H}$-bonding aggregates (precursors of the crystalline structure) generating super-dipole moments.
\end{abstract}




\section{Introduction}

Glass forming materials are omnipresent in our daily life and play an essential role in modern technology. Their physical understanding is still controversial and remains an unsolved problem of condensed matter physics. ${ }^{1,2}$ One of the most intriguing phenomenon is the extraordinary increase of the structural relaxation time in the supercooled liquid state when approaching the glass transition temperature $\left(T_{\mathrm{g}}\right)$, the non-Debye character of the frequency or time domain response and non-Arrhenius behavior of the temperature dependence. ${ }^{3-8}$ The dynamical behavior of glass-forming liquids and thus of their relaxational processes cover a wide range of time scales from local vibrations to the highly cooperative motions as the temperature approaches $T_{g} .{ }^{9}$ When it comes to molecular mobility investigation in glass forming liquids or disordered systems exhibiting a permanent dipole moment, Dielectric Relaxation Spectroscopy (DRS) remains one of the most powerful and useful tool to achieve this goal. ${ }^{10-17}$

Generally, for most glass forming liquids, the main process localized at the lowest frequency is recognized as the structural relaxation process ( $\alpha$-relaxation) associated to the dynamic of glass transition and molecular cooperative motions in an environment of few nanometers. ${ }^{7,18-}$ 20 The frequency and temperature dependency of dielectric features of the $\alpha$-relaxation present a non-Debye and non-Arrhenius character respectively. However, some glass formers display a slower dielectric relaxation than $\alpha$-process with a Debye-like behavior and a nonArrhenius temperature dependency of the relaxation times.

The so-called D-process has been observed in many hydrogen-bonded liquids such as water, $^{21,22}$ monohydroxy alcohols, ${ }^{9,23-28}$ secondary amides $\mathrm{N}$-mono-substituted amides, ${ }^{29-32}$ and pharmaceuticals. ${ }^{12,33-35}$

Furthermore, for monohydroxy alcohols and secondary amides, the dielectric strength usually exceeds by several orders of magnitude the expected value predicted from the molecular dipole moment basis. ${ }^{23,29}$ Surprisingly, the D-process does not contribute in the calorimetric signature ${ }^{36,37}$ although a very intense dielectric response. Likewise, viewed from earlier shear mechanical spectroscopy experiments, no significant contribution in the mechanical shear signal was associated to D-process. Since then, Gainaru et $a^{38}$ evidenced by high resolution shear mechanical spectroscopy the mechanical signature of D-process in two monohydroxy 
alcohols close to the glass transition region. Moreover, the rheological behavior is similar to what is observed for short chain polymers. ${ }^{38}$ Nevertheless, the origin of the D-process has been a matter of discussions for many decades and still remains an unsolved puzzle in condensed matter physics. ${ }^{24,39-41}$ In $\mathrm{H}$-bonded liquids, the D-process often originates from structural correlation between several dipoles. ${ }^{42}$ Numerous models have been proposed to explain the microscopic origin of the D-process in these systems: wait and switch models of dipole reorientation ${ }^{25,43,44}$ (breaking of $\mathrm{H}$-bonds in a chain and formation of a new chain leading to end-to-end fluctuations of the dipole moment), dipole inversion by cooperative rotation of the $\mathrm{OH}$-groups ${ }^{45}$ and self-restructuring transient-chain model, where molecules break off from or add to the ends of the chain promoting slow rotations of the effective dipoles of the chain. ${ }^{46}$ So far, none of these models can give a universal account of the origin of the Debye behavior. Thus, a more fundamental and predictive model is still expected..$^{42}$ Nevertheless, the postulated models figure out the important role played by $\mathrm{H}$-bonds in the expression of D-process.

In order to shed some light on the microscopic origin of the peculiar D-process, we have performed a thorough combined investigation of physical properties of an $\mathrm{H}$-Bond (HB) glass forming liquid, $\mathrm{N}$-acetyl- $\alpha$-methylbenzylamine, $\mathrm{C}_{10} \mathrm{H}_{13} \mathrm{NO}$ (Figure 1 ), by means of Dielectric Relaxation Spectroscopy (DRS), Temperature Modulated Differential Scanning Calorimetry (TMDSC), Fast Scanning Calorimetry (FSC), and Molecular Dynamics (MD) Simulations. Nacetyl- $\alpha$-methylbenzylamine (Nac-MBA hereafter), is a chiral mono-substituted amide derivative from $\alpha$-methylbenzylamine (a widely used chiral resolving agent for optical resolution procedures ${ }^{47}$ ).

In this study the investigated Nac-MBA system (Figure 1 ) is actually a mixture of $25 \%$ of the pure $\mathrm{R}(+)$ enantiomer with $75 \%$ of the counter $\mathrm{S}(-)$ enantiomer, since the optically pure enantiomers of Nac-MBA present an enhanced crystallization tendency (forthcoming paper). 


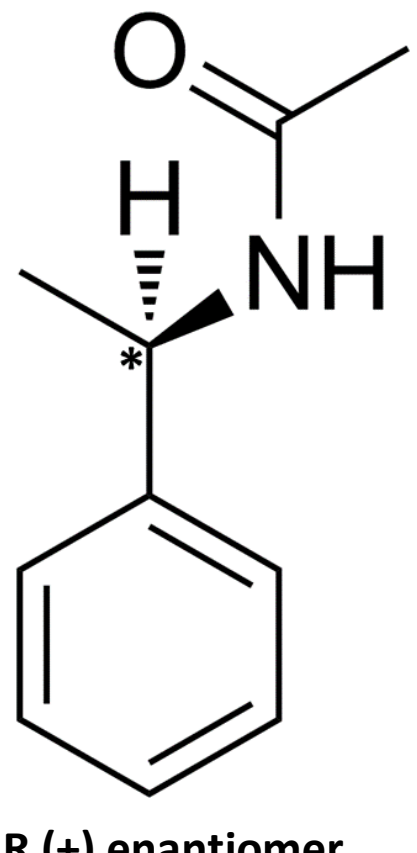

$R(+)$ enantiomer

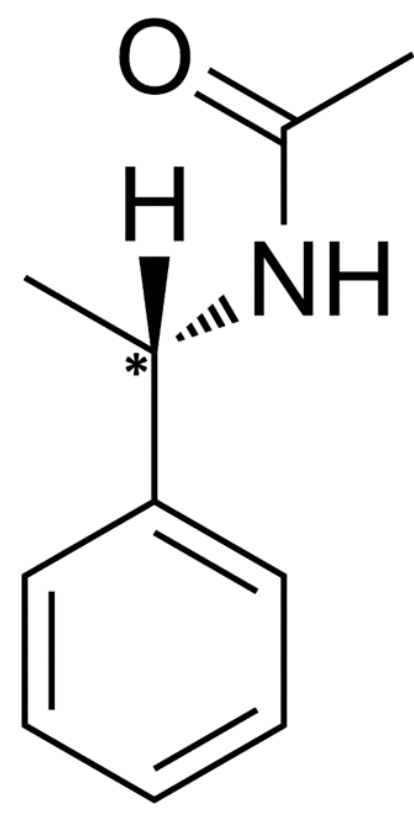

$S(-)$ enantiomer

Figure 1: Developed formula of Nac-MBA enantiomers.

\section{Materials and methods.}

\subsection{Synthesis of $R(+)$ and S(-) Nac-MBA}

Optically pure $\mathrm{R}(+)$ or $\mathrm{S}(-)$ Nac-MBA was obtained by acetylation of $\mathrm{R}(+)$ or $\mathrm{S}(-)$ $\alpha$-methylbenzylamine purchased from Aldrich (99\%) using acetyl chloride. ${ }^{48,49}$ The method (see ref ${ }^{50}$ for more details) consists to dilute the amine in dichloromethane and mixed the diluted solution with an aqueous solution of sodium carbonate initially prepared. Slight excess of diluted acetyl chloride in dichloromethane is slowly added to the previous solution. After stirring, the reaction mixture is decanted and the unreacted products are removed by successive washings with acid and basic aqueous solutions. The washed solution is dried with $\mathrm{MgSO}_{4}$ and evaporated (yield almost $90 \%$ ). The white solid obtained is doubly recrystallized from toluene or purified by column chromatography. ${ }^{50}$

The mixture studied in the present work $(25 \% \mathrm{R}(+)$ plus $75 \% \mathrm{~S}(-))$, corresponds to an enantiomeric excess, ee (i.e. the absolute difference between the mole fractions of each enantiomer) of $50 \%$. 


\subsection{Temperature Modulated Differential Scanning Calorimetry (TMDSC)}

TMDSC and conventional DSC analyses were carried out with a Q100 DSC from TA instruments equipped with a refrigerated cooling system allowing a cooling down to $203 \mathrm{~K}$. All measurements were performed under nitrogen gas flow of $50 \mathrm{~mL} / \mathrm{min}$. Baseline (empty cell) was calibrated at a chosen heating rate from 203 to $423 \mathrm{~K}$. Temperature and enthalpy calibrations were achieved using indium and benzophenone as reference materials. An empty aluminum pan, identical to that used for the sample, was used as reference. Specific heat capacity was calibrated using two sapphire discs as a reference materials.

TMDSC experiments were carried out in the "heat only" modulation mode with 1) an oscillation amplitude of $\pm 0.318 \mathrm{~K}$ and oscillation period of $60 \mathrm{~s}$, during heating at $2 \mathrm{~K} / \mathrm{min}$ and 2) an oscillation amplitude of $\pm 1 \mathrm{~K}$ and oscillation period of $60 \mathrm{~s}$ during heating at $0.5 \mathrm{~K} / \mathrm{min}$. The oscillation period refers to the time needed to complete one temperature modulation cycle.

The modulation scanning was performed on an initially amorphous sample obtained during a previous cooling at $70 \mathrm{~K} / \mathrm{min}$ carried out on the conventional mode.

Sample Preparation: In order to prepare the DSC samples with an enantiomeric excess of $50 \%$ in the S(-) enantiomer, $75 \mathrm{mg}$ of S(-) Nac-MBA and $25 \mathrm{mg}$ of $\mathrm{R}(+)$ Nac-MBA were manually grounded in a mortar and $5 \mathrm{mg}$ of this physical mixture was encapsulate in a $\mathrm{T}_{\text {zero }}$ aluminum pan. Each experiment was repeated at least twice using fresh samples.

\subsection{Fast Scanning Calorimetry (FSC)}

FSC measurements were conducted with a power compensation twin-chip namely Flash DSC 1 (FSC) from Mettler-Toledo. Cooling was ensured down to $203 \mathrm{~K}$ by an intracooler model Huber TC100. Approximately $35 \mathrm{ng}$ of sample was loaded onto the central position of a sensor chip using an optical microscope and a long fiber for sample positioning. The sample was submitted to a nitrogen purge gas flow at a rate of $20 \mathrm{~mL} / \mathrm{min}$. Successive conditioning and corrections of the chip sensor were performed according to the manufacturer and prior to measurements. ${ }^{51}$ As highlighted in previous work on FSC, ${ }^{52}$ it remains ambiguous to ensure an effective composition of ee $=50 \%$ even though the sampling (dozens of $\mathrm{ng}$ ) were taken from a $10 \mathrm{mg}$ mixture of both enantiomers in such way that the average ee $=50 \%$. Here, we 
considered the ee as intermediate between enantiomerically pure and racemic composition (i.e. scalemic). Sample mass was estimated by comparing the amplitude of the heat capacity step at the glass transition with values obtained from classical DSC. A pre-melting step was launch to ensure homogeneous distribution of the sample on the chip area. With regards to determine the cooling rate dependency of the fictive temperature $T_{f}$, a cyclic heat-cool thermal program was applied. This protocol consisted in quenching the liquid at different cooling rates from $1500 \mathrm{~K} / \mathrm{s}$ down to $5 \mathrm{~K} / \mathrm{s}$ followed by a heating scan at $1500 \mathrm{~K} / \mathrm{s}$. To ensure reproducibility, all measurements were performed at least twice.

\subsection{Dielectric Relaxation Spectroscopy (DRS)}

\section{a - Low frequency range $0.1 \mathrm{~Hz}-1 \mathrm{MHz}$}

Dielectric measurements where performed using high quality interdigitated gold plated electrodes, IE (BDS1410-20-150) purchased from Novocontrol GmbH. The electrode is a round shape $20 \mathrm{~mm}$ diameter gold plated copper layers with $35 \mu \mathrm{m}$ thickness and a comb structure for convenient sample attachment. The spacing between the comb fingers and their width are both $150 \mu \mathrm{m}$. Prior to sample deposition, the electrode was calibrated by measuring its respective geometric (empty cell) capacity, $C_{0}$, and substrate capacity, $C_{\text {su, }}$ through measurement of a standard material with known permittivity (B-oil; Vacuubrand). As the substrate material of the IE has a low loss factor $(\tan \delta=0.001)$ and the substrate real part permittivity being approximately constant over frequency and temperature, the calibration procedure ${ }^{53}$ can be simplified if the permittivity of the electrode substrate $\varepsilon_{s u}^{*}$ is approximated by a real constant $\varepsilon_{s u}$. For a selected frequency, $f($ e.g. $10 \mathrm{kHz})$, the capacity of the empty electrode without sample material attached $C_{e}^{*}(f)$ and the capacity of the electrode with the calibration oil attached $C_{c}^{*}$ (permittivity $\varepsilon_{S}^{*}$ ) are measured. The total capacity is determined considering that the upper and lower electrodes constitute two independent volumes generating two independent capacitors ${ }^{54,55}$ with a total capacity of:

$$
C_{m}^{*}=C_{0}\left(\varepsilon_{s}^{*}(f)+\varepsilon_{s u}(f)\right)
$$

with

$$
C_{0}=\frac{C_{c}^{*}(f)-C_{e}^{*}(f)}{\varepsilon_{c}^{*}(f)}
$$

The complex frequency-dependent permittivity $\varepsilon^{*}(f)=\varepsilon^{\prime}(f)-i \varepsilon^{\prime \prime}(f)$ (where $\varepsilon^{\prime}=$ storage permittivity and $\varepsilon^{\prime \prime}=$ loss permittivity) was measured over the range $10^{-1} \mathrm{~Hz}-2 \mathrm{MHz}$ using an 
Alpha-A analyzer from Novocontrol Technologies $\mathrm{GmbH}$. Sample preparation and amorphization steps were performed ex situ. Almost $150 \mathrm{mg}$ of the crystalline powder was deposited on the IE then heated above the liquidus temperature at $383 \mathrm{~K}$ and annealed 5 minutes to guarantee complete melting. The melt was then promptly quenched in liquid nitrogen in order to ensure total amorphization (according to previous work by some of us on the amorphization conditions of Nac-MBA). ${ }^{50}$ Dielectric spectra were collected isothermally from $153 \mathrm{~K}$ up to $273 \mathrm{~K}$ with a suitable consecutive step: measurements were performed from $241 \mathrm{~K}$ to $273 \mathrm{~K}$ with an increment of $1 \mathrm{~K}$; for $\mathrm{T}<241 \mathrm{~K}$ an increment of 2 or $5 \mathrm{~K}$ was considered.

\section{b - High frequency range $1 \mathrm{MHz}-1 \mathrm{GHz}$}

Measurements were performed in the range $1 \mathrm{MHz}-1 \mathrm{GHz}$ by mean of a coaxial line reflectometer based on the impedance analyzer HP 4191 from Novocontrol Technologies $\mathrm{GmbH}$. Preceding measurements, two successive calibration steps where achieved: Line and sample cell calibrations. Samples were prepared in a parallel plate geometry between two gold-plated electrodes of $10 \mathrm{~mm}$ diameter, separated by silica spacers of $50 \mu \mathrm{m}$ thickness. The prepared sample cell was connected to the end of the coaxial line, then melted in situ and annealed for 5 minutes at $383 \mathrm{~K}$. Isothermal frequency scans were carried out upon cooling from $383 \mathrm{~K}$ down to $273 \mathrm{~K}$, by steps of $10 \mathrm{~K}$.

A Quatro Cryosystem (Novocontrol Technologies $\mathrm{GmbH}$ ) was used to control the temperature with a stability of $\pm 0.2 \mathrm{~K}$ for both low and high frequency domains.

\section{c - Data Analysis}

In order to access the time scale of each of the four processes expressed in Nac-MBA (ee=50\%) system, their temperature dependencies, the shape parameters, and the dielectric strength, the isothermal dielectric spectra $\left(\varepsilon^{\prime}\right.$ and $\left.\varepsilon^{\prime \prime}\right)$ were fitted with a sum of Havriliak-Negami model functions ${ }^{56}$ and a conductivity contribution for $\varepsilon^{\prime \prime}$, writing as followed:

$$
\varepsilon^{*}(\omega)=\varepsilon^{\prime}(f)-i \varepsilon^{\prime \prime}(f)=\varepsilon_{\infty}+\sum_{k}^{n}\left[\frac{\Delta \varepsilon_{k}}{\left(1+i\left(2 \pi f \tau_{H N_{k}}\right)^{\alpha_{H N_{k}}}\right)^{\beta_{H N_{k}}}}\right]-i\left(\frac{\sigma_{d c}}{2 \pi f \varepsilon_{0}}\right)^{N}
$$

where $\sigma_{d c}$ is the specific dc-conductivity; $\varepsilon_{0}$ the permittivity of the vacuum $\left(\varepsilon_{0}=\right.$ $\left.8.854 \times 10^{-12} \mathrm{~F} / \mathrm{m}\right) ; f$ the frequency; $N$ gives the exponent of the frequency dependence of 
$\varepsilon^{\prime \prime} ; k$ sums over the different relaxation processes, $\tau_{H N}$ is a characteristic relaxation time regards to the frequency of maximal loss $f_{\max } ; \Delta \varepsilon$ is the dielectric relaxation strength of the process under investigation; $\varepsilon_{\infty}$ the high frequency limit of the real permittivity $\varepsilon^{\prime}(f) . \alpha_{H N}$ and $\beta_{H N}$ are fractional shape parameters which detail the symmetric and asymmetric broadening of the complex dielectric response in such a way that $0<\alpha_{H N}, \alpha_{H N} \cdot \beta_{H N} \leq 1 .{ }^{10}$ From the derived values of $\tau_{H N}, \alpha_{H N}, \beta_{H N}$, a model free relaxation time $\tau$ of each relaxation process is given by:

$$
\tau=\frac{1}{2 \pi f_{\max }}=\tau_{H N}\left[\sin \left(\frac{\alpha_{H N} \pi}{2+2 \beta_{H N}}\right)\right]^{\frac{-1}{\alpha_{H N}}}\left[\sin \left(\frac{\alpha_{H N} \beta_{H N} \pi}{2+2 \beta_{H N}}\right)\right]^{\frac{1}{\alpha_{H N}}}
$$

\subsection{Molecular Dynamics Simulations (MD)}

Molecular dynamics (MD) simulations have been performed using the DL_POLY package ${ }^{57}$ and the GAFF force field (General Amber Force Field). ${ }^{58}$ This force field was chosen due to its capability of reproducing successfully large number of experimental data of low molecular weight molecules. ${ }^{59}$ As recommended for the GAFF force field, ${ }^{58}$ atomic partial charges were calculated from ab-initio computations using the Gaussian 03 programme ${ }^{60}$ at the HF/6-31G* level with the restrained electrostatic potential (RESP) fitting approach. For the electrostatic interactions, a pairwise damped shifted method developed by Wolf ${ }^{61}$ was used. For both van der Waals and electrostatic interactions, the same cutoff radius of $10 \AA$ was employed. Periodic boundary conditions were applied in all directions. The time step to integrate Newton's equation of motion was chosen to $0.001 \mathrm{ps}$. The modelled system possesses a total number of $\mathrm{N}=64$ molecules composed of $48 \mathrm{~S}(-)$ Nac-MBA and $16 \mathrm{R}(+)$ Nac-MBA molecules in order to obtain an enantiomeric excess of $50 \%$. Simulations have been conducted either in the NPT or NVT statistical ensemble where $\mathrm{P}$ is the pressure, $\mathrm{T}$ the temperature and $\mathrm{V}$ the volume. Pressure and temperature were controlled with a Nose-Hoover barostat and thermostat respectively. All NPT simulations were realized at atmospheric pressure. The Nosé Hoover thermostat and barostat relaxation times have been set at 0.2 and 2.0 ps respectively. The equilibrated volume of the simulation box during the NPT simulation was considered to compute the averaged density of system and used to perform the subsequent production simulation in the NVT ensemble. MD simulations were performed at five temperatures $500 \mathrm{~K}$, $450 \mathrm{~K}, 400 \mathrm{~K}, 360 \mathrm{~K}$ and $340 \mathrm{~K}$. The equilibration/production times range from 10/200 ns to 
20/800 ns from the highest to the lowest investigated temperature. The Nac-MBA densities determined in the present study are shown in figure 2 . The monotonic behavior of the density as function of the temperature confirms that the system is equilibrated over the simulated times. The calculated density of the liquid extrapolated to a low temperature near below the glass transition temperature $\left(\rho(235 \mathrm{~K})=1.1 \mathrm{~g} / \mathrm{cm}^{3}\right)$ is consistent regarding the crystalline counterpart: $\rho=1.13 \mathrm{~g} / \mathrm{cm}^{3} .{ }^{47}$ Indeed, typically, the density of the glassy state is above $90 \%$ of the density of the $\operatorname{crystal}^{62-64}\left(\right.$ i.e. $\left.\rho=1.017 \mathrm{~g} / \mathrm{cm}^{3}\right)$.

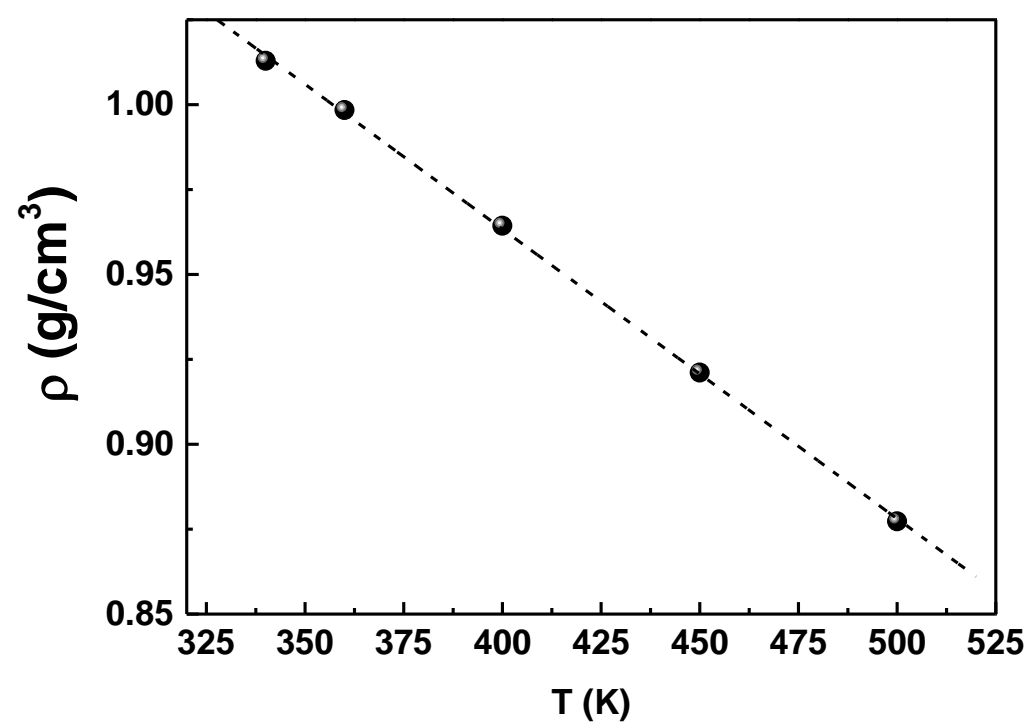

Figure 2: Temperature evolution of the density of Nac-MBA (ee=50\%) determined from MD simulations. 


\section{Results and discussions}

\subsection{Thermal behavior characterization of Nac-MBA by TMDSC and FSC}

Figure 3a) shows conventional DSC thermograms of a physical mixture of Nac-MBA (ee $=50 \%$ ) for the first (curve 1) and the second (curve 2) heating scans at $2 \mathrm{~K} / \mathrm{min}$. When heating the crystalline powder mixture, three successive endotherms are evidenced and associated to the eutectoid $\left(T_{\text {eutectoid }}=338 \mathrm{~K}\right)$, eutectic $\left(T_{e}=348 \mathrm{~K}\right)$ and liquidus $\left(T_{L}=353 \mathrm{~K}\right)$ phenomena, respectively. At the eutectoid temperature, the physical mixture of both enantiomers (conglomerate) converges towards a co-crystal of both enantiomers (racemic compound). These results are in good agreement with the phase diagram between both enantiomers of Nac-MBA proposed in the work of Druot et al. ${ }^{47}$ During liquid quenching from $383 \mathrm{~K}$ down to $203 \mathrm{~K}$ at circa $70 \mathrm{~K} / \mathrm{min}$ (not shown) no sign of crystallization is observed. The subsequent heating scan (curve 2) shows the characteristic heat flow step for the calorimetric glass transition at circa $240 \mathrm{~K}$ along with the typical enthalpy recovery peak (zoom in insert). It is a proof that an amorphous glass has been obtained during the earlier stage of melt quenching. Therefore, under this condition, Nac-MBA can be considered as a good glass forming liquid. Upon further heating well above $T_{g}$, cold-crystallization of the supercooled liquid is evidenced at $\mathrm{T}_{\mathrm{Cr}}{ }^{\text {n }}=273 \mathrm{~K}\left(\Delta \mathrm{H}_{\mathrm{cr}}=70 \pm 5 \mathrm{~J} / \mathrm{g}\right)$. This exothermal peak is followed by a double endothermal event related to the eutectic and liquidus events of the corresponding racemic compound. No thermal event revealed the existence of the conglomerate equilibrium. Consequently, from the amorphous glassy state Nac-MBA (ee=50\%) evolves towards metastable equilibrium.

TMDSC allows to measure the heat capacity and kinetic components (usually referred as reversing and nonreversing components) of the total heat flow signal obtained in conventional DSC. ${ }^{65}$ Figure $\left.3 b\right)$ depicts the reversing specific heat capacity obtained for amorphous NacMBA upon heating at $2 \mathrm{~K} / \mathrm{min}$ : the thermal glass transition region is very narrow with onset temperature $T_{g}^{\text {onset }}=240.7 \mathrm{~K}$ and $\mathrm{T}_{\mathrm{g}}$ determined from the mid-step position $T_{g}^{\text {mid }}=242.4$ $\mathrm{K}$. The heat capacity change at $\mathrm{T}_{\mathrm{g}}$ is $\Delta \mathrm{C}_{\mathrm{P}}=0.45 \pm 0.01$ (J/(g.K). It is worth pointing out that while the crystallization ability is strongly impacted by the enantiomeric concentration (forthcoming communication), the glass transition temperature and $\Delta C_{p}$ are independent of the enantiomeric excess. ${ }^{50}$ 

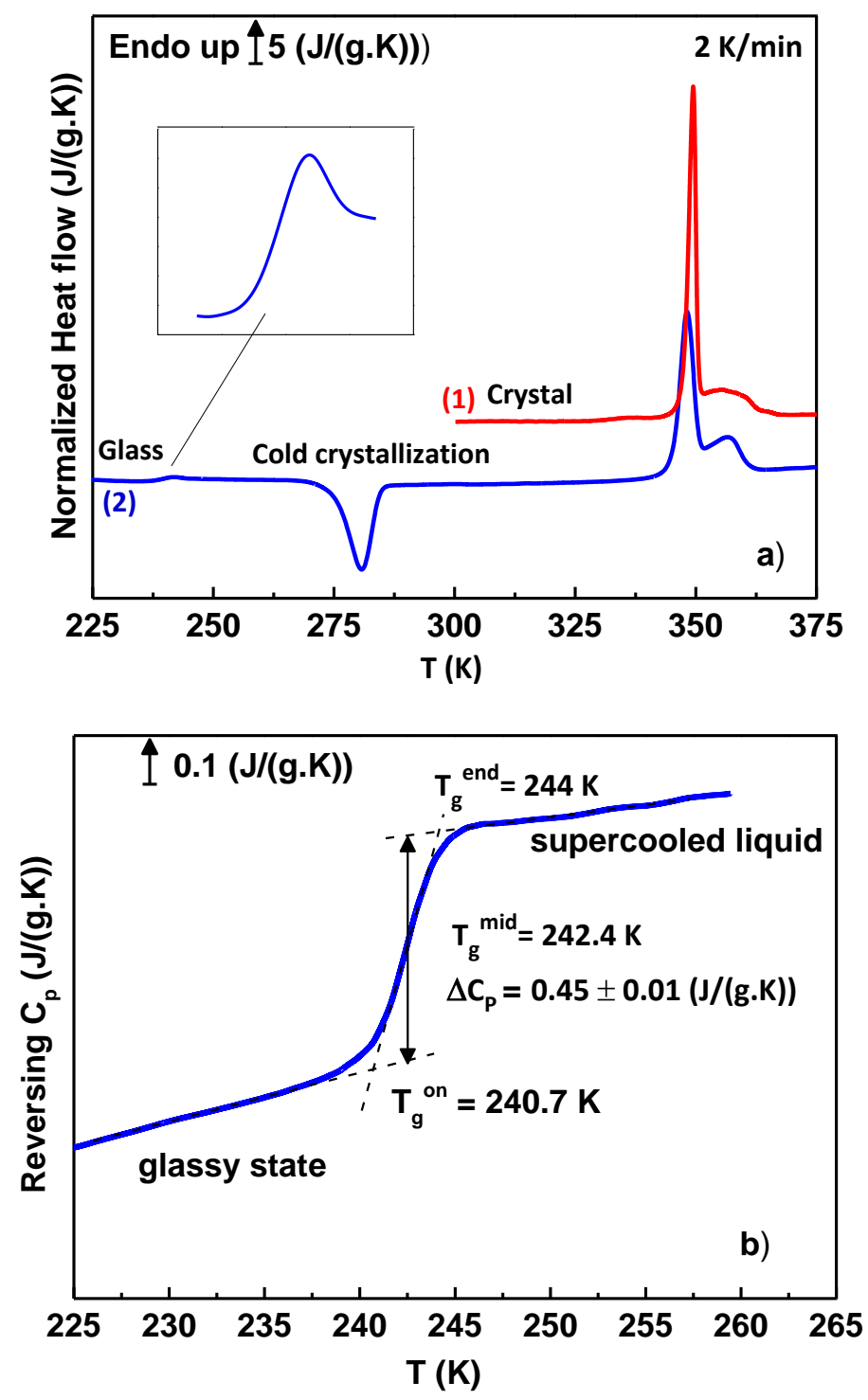

Figure 3: a) Thermal behavior (normalized total heat flow versus temperature) of Nac-MBA (ee=50\%) during heating at $2 \mathrm{~K} / \mathrm{min}$ (standard DSC); b) Reversing specific heat capacity $\left(C_{p}\right)$ as function of temperature (at $2 \mathrm{~K} / \mathrm{min}$ ) in the glass transition domain (TMDSC).

Fast scanning calorimetry is used to characterize the glass transition in terms of the variation of the fictive temperature over a wide range of cooling rates. The fictive temperature concept was introduced by Tool and Eicitlin ${ }^{66}$ and defines the temperature at which any properties related to the structural relaxation are at the equilibrium state. Figure 4 depicts the temperature evolution of the normalized heat flow during heating at rate $q_{h}=1500 \mathrm{~K} / \mathrm{s}$, after several cooling scans with a rate $\left(q_{c}\right)$ ranging from $5 \mathrm{~K} / \mathrm{s}$ up to $1500 \mathrm{~K} / \mathrm{s}$. The enthalpy recovery peak observed at the glass transition region shifts towards higher temperatures and 
its magnitude increases with decreasing cooling rate as classically observed in literature for physical aging phenomena (for increasing aging time). ${ }^{67-69}$ When $q_{c}=q_{h}=1500 \mathrm{~K} / \mathrm{s}, T_{g}$ is shifted toward higher temperatures for more than $10 \mathrm{~K}$ compared to standard DSC results obtained for $q_{h}=2 \mathrm{~K} / \mathrm{min}=0.033 \mathrm{~K} / \mathrm{s}$. This shift is the expression of the kinetical character of the glass transition phenomenon observed for such kind of thermal protocol. ${ }^{70}$ The fictive temperature $\left(T_{f}\right)$ calculation was performed according to the equal area rule method ${ }^{71}$ (details on $T_{f}$ calculation procedure are described in ref $^{52}$ ). The change in the fictive temperature at variable cooling rates can be used to describe the temperature dependence of the structural $\alpha$-relaxation. ${ }^{67,72,73}$ The empirical approach proposed by Frenkel-KobekoReiner $(\mathrm{FKR})^{72}$ illustrates the link between the cooling rate $q_{c}(\mathrm{~K} / \mathrm{s})$ and the structural relaxation time $\tau(\mathrm{s})$ express as follow:

$$
\boldsymbol{q}_{\boldsymbol{c}} \boldsymbol{\tau}=\boldsymbol{C}
$$

where $C$ (Kelvin) is a shift factor usually assume to be a constant ${ }^{67,74}$. Consequently it should be considered that:

$$
\log \left(\boldsymbol{q}_{c}\right)=-\log (\tau)+\log (\boldsymbol{C}) \quad \text { (Eq. 6) }
$$

This proposal is generally used to combine DSC and/or FSC data with features of the dynamic glass transition as probed from heat capacity or dielectric relaxation spectroscopy measurements. ${ }^{67,74-77}$ Superposition of the temperature dependence of the cooling rate and the dielectric $\alpha$-relaxation time will be reported in the relaxation map (Figure 9a)); section 3.2.2).

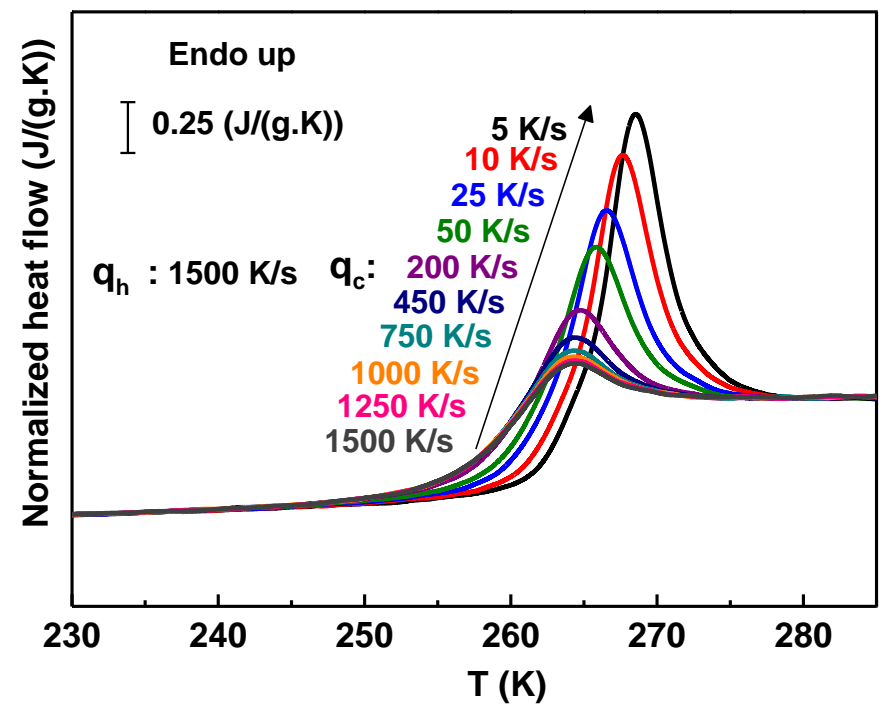


Figure 4: FSC heating curves of Nac-MBA obtained at $q_{h}=1500 \mathrm{~K} / \mathrm{s}$ after cooling from the supercooled liquid state down to the glassy state at cooling rate $q_{c}$ ranging from $5 \mathrm{k} / \mathrm{s}$ to $1500 \mathrm{~K} / \mathrm{s}$.

\subsection{Molecular Mobility of Nac-MBA (ee $=50 \%$ ) probed by DRS}

As highlighted by calorimetric results, amorphous glass Nac-MBA (ee=50\%) can be obtained by melt quenching. For dielectric characterization of the glassy and supercooled liquid state near and above $T_{g}$, the amorphous sample was prepared ex-situ to guarantee a sufficient high cooling rate in order to avoid crystallization. Measurements of the complex permittivity $\varepsilon^{*}(f)$ were then performed isothermally, during heating, in the temperature range [153 K, $273 \mathrm{~K}$ ] with a frequency scan from $0.1 \mathrm{~Hz}$ to $2 \mathrm{MHz}$ for each selected temperature. Isothermal, high frequency $(1 \mathrm{MHz}-1 \mathrm{GHz})$ measurements of $\varepsilon^{*}(\mathrm{f})$ were carried out when cooling the liquid from $383 \mathrm{~K}$ down to $283 \mathrm{~K}$ with regards to access the molecular mobility at higher temperatures in the supercooled and liquid state (see experimental section 2.4 for details).

\subsubsection{Identification and analysis of dipolar relaxations}

The temperature dependence of the real permittivity $\varepsilon^{\prime}$ at some representative frequencies (taken from the isothermal dielectric data acquired upon heating) is shown in Figure 5a). Near the calorimetric glass transition a frequency dependent step increase is observed. For temperatures higher than $260 \mathrm{~K}$ a steep decrease is visible in the $\varepsilon^{\prime}(T)$. This drop in the dielectric permittivity is frequency independent and represents the manifestation of coldcrystallization. Melt crystallization was also observed at circa $273 \mathrm{~K}$ during the isothermal high frequency (RF) measurements (not shown). Therefore, in the following only the dielectric spectra obtained for the fully amorphous samples will be taken for analysis and discussion (i.e., data taken at $260 \mathrm{~K}<\mathrm{T}<283 \mathrm{~K}$ are excluded). As shown in the inset of figure $5 \mathrm{a}$ ), a very intense dielectric loss $\varepsilon^{\prime \prime}$ response is observed in the supercooled liquid, associated to the frequency dependent step $\varepsilon^{\prime}$ increase when the sample is heated from the glassy state. A close up of the $\varepsilon^{\prime \prime}(T)$ trace at the lowest measured frequency $(0.1 \mathrm{~Hz})$ is depicted in Figure $\left.5 \mathrm{~b}\right)$. A quick analysis of this spectrum using two Gaussian functions allows clear evidence for two relaxations: a very low intensity peak with maximum at $T_{\max }=240.9 \mathrm{~K}$ and a predominant one at $T_{\max }=246.3 \mathrm{~K}$. For further information on these processes, Figure $5 \mathrm{c}$ ) shows the first 
temperature derivative of the heat capacity $C_{p}{ }^{\prime}(T)$ curve. The real part of the complex heat capacity $\left(C_{p}{ }^{\prime}\right)$ of a system is related to the reversing heat capacity measured from TMDSC. ${ }^{15,78,79}$ The $C_{p}{ }^{\prime}$ curve shown in the inset of figure $5 c$ ) was obtained by TMDSC during heating at $0.5 \mathrm{~K} / \mathrm{min}$ with an oscillation period of $60 \mathrm{~s}\left(\mathrm{f}=1.7 \times 10^{-2} \mathrm{~Hz}\right)$. The use of such scanning conditions allow a thermal treatment (and time scale) that reasonably compares to the dielectric relaxation experiments (equilibrium isothermal measurements were performed with temperature steps that correspond to an average heating rate of around $0.3 \mathrm{~K} / \mathrm{min}$ ). The temperature derivative of $C_{p}{ }^{\prime}(T)$ curve (proportional to the imaginary component of the heat capacity, $\left.C_{p}{ }^{\prime \prime}\right)$ is perfectly described by a single Gaussian function with a full width at half maximum $(\mathrm{FWHM})$ of $\sim 3.9 \mathrm{~K}$ and centered at $\mathrm{T}_{\max }=240.4 \mathrm{~K}$. This temperature corresponds to the middle temperature of the sigmoidal shaped $C^{\prime}{ }_{p}(T)$, i.e., the kinetic glass transition temperature $\left(\mathrm{T}_{\mathrm{g}}{ }^{\text {mid }}=240.4 \pm 0.5 \mathrm{~K}\right)$. The low temperature shoulder in the $\varepsilon^{\prime \prime}(\mathrm{T})$ trace $\left(\mathrm{T}_{\max }=\right.$ $240.9 \mathrm{~K} ; \mathrm{FWHM}=3 \mathrm{~K}$ ) is clearly associated with the measurable change of heat capacity and therefore it is recognized as the signature of the structural (primary) $\alpha$-relaxation. In spite of its high dielectric magnitude, the high temperature relaxation $\left(T_{\max }=246.3 \mathrm{~K}, \mathrm{FWHM}=5 \mathrm{~K}\right.$ ) shows no calorimetric activity. Similar features are observed for hydrogen bonded liquids where a dominant Debye-like process (D-process) slower than the smaller $\alpha$-relaxation is observed. ${ }^{31,32,38,41}$

For the Nac-MBA system here studied the high amplitude relaxation is also identified as a Dprocess taking into account that its dielectric loss frequency dependency can be well described by a single Debye function as it is illustrated later on (Figure $7 b$ ). 

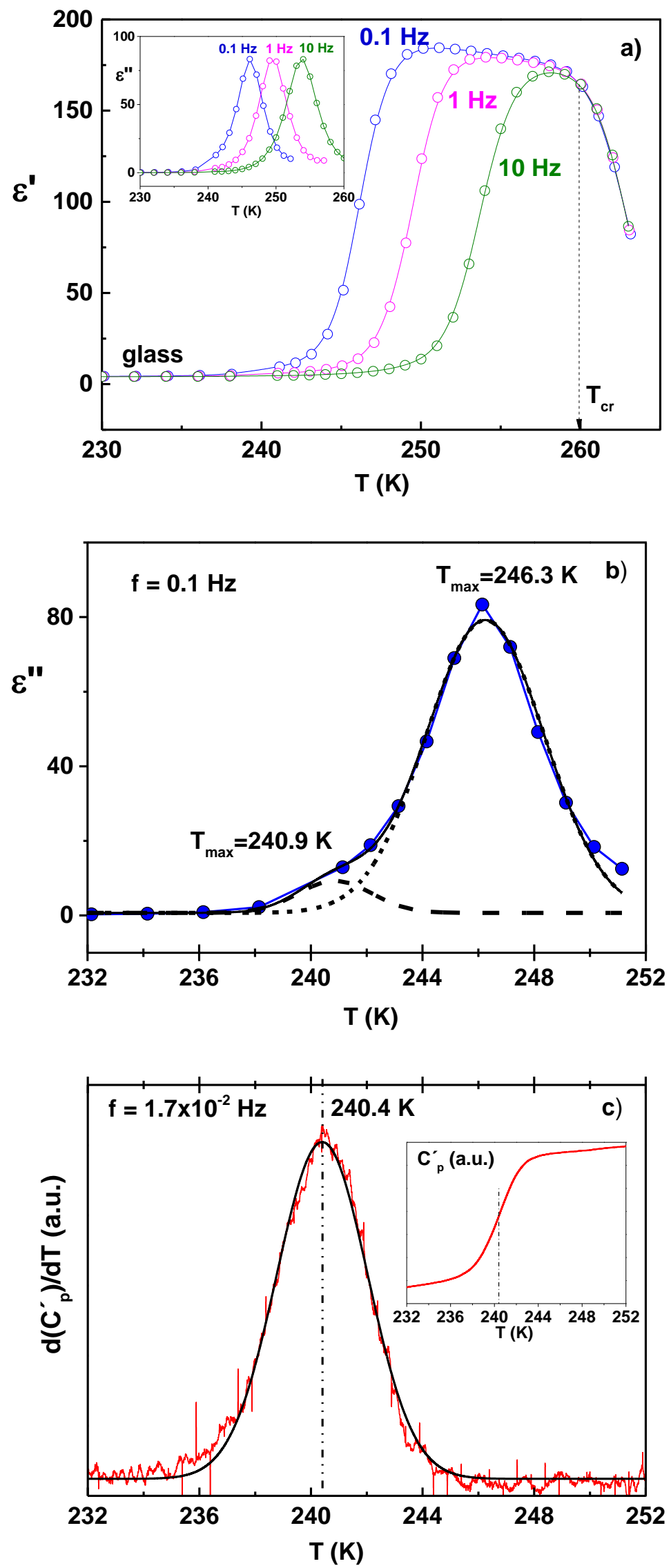
Figure 5: a) Temperature dependence of the real part of complex permittivity $\left(\varepsilon^{\prime}\right)$ and dielectric loss $\varepsilon^{\prime \prime}$ (insert) of Nac-MBA (ee $=50 \%$ ) for the indicated frequencies. b) $\varepsilon^{\prime \prime}(T)$ for $f$ $=0.1 \mathrm{~Hz}$ (circles) in the glass transition temperature range [232 K-252 K]. Solid line - global fit using two Gaussian functions (dashed lines). c) First temperature derivative $\left(d\left(C_{p}^{\prime}\right) / d T\right)$ of the $C_{p}{ }^{\prime}$ curve shown in inset ( $\sim$ reversing heat capacity obtained by TMDSC upon heating at $0.5 \mathrm{~K} / \mathrm{min}$ with temperature oscillation period $60 \mathrm{~s}=1.7 \times 10^{-2} \mathrm{~Hz}$ ). Solid line: Gaussian curve fit to $d\left(C_{p}{ }^{\prime}\right) / d T$ data.

Figure 6a) depicts the dielectric loss spectra as a function of the frequency for Nac-MBA at given temperatures below the glass transition temperature. The asterisks are visual guides delimiting three clearly distinct dispersion regions labelled $\alpha-, \beta$ - and " $\gamma$ "-, in order of increasing frequency. As expected, at temperatures close to the dynamic glass transition $\left(\mathrm{T}_{\mathrm{g}}{ }^{\text {mid }}\right.$ $=240 \mathrm{~K} ; \mathrm{f} \sim 2 \times 10^{-2} \mathrm{~Hz}$ ) the high frequency tail of the $\alpha$-relaxation becomes visible in the available frequency range. At higher frequencies than the $\alpha$-relaxation, a secondary $\beta$-process appears as a shoulder that clearly shift towards higher frequencies as temperature increases. Deep in the glassy state, the $\beta$-relaxation seems to leave the frequency range and only a very broad dispersion (" $\gamma$ "-relaxation) remains. Figure $6 b$ ) shows the isochronal plot of $\log \left(\varepsilon^{\prime \prime}\right)$ at chosen frequencies that allows a further evidence for the appearance of this broad " $\gamma$ "relaxation at lower temperatures than the $\beta$-relaxation (see also the close up in insert). For frequency $f=5 \times 10^{3} \mathrm{~Hz}$ a crossover between the straight lines drawn to qualitatively describe the temperature dependence of $\varepsilon^{\prime \prime}(\mathrm{T})$ of the $\beta$ - and $\alpha$-process occurs at circa $240 \mathrm{~K}$, close to the glass transition temperature, $\mathrm{T}_{\mathrm{g}}{ }^{\text {mid }}$. This feature is an indication of a Johari-Goldstein $\beta$ relaxation. ${ }^{80}$ 

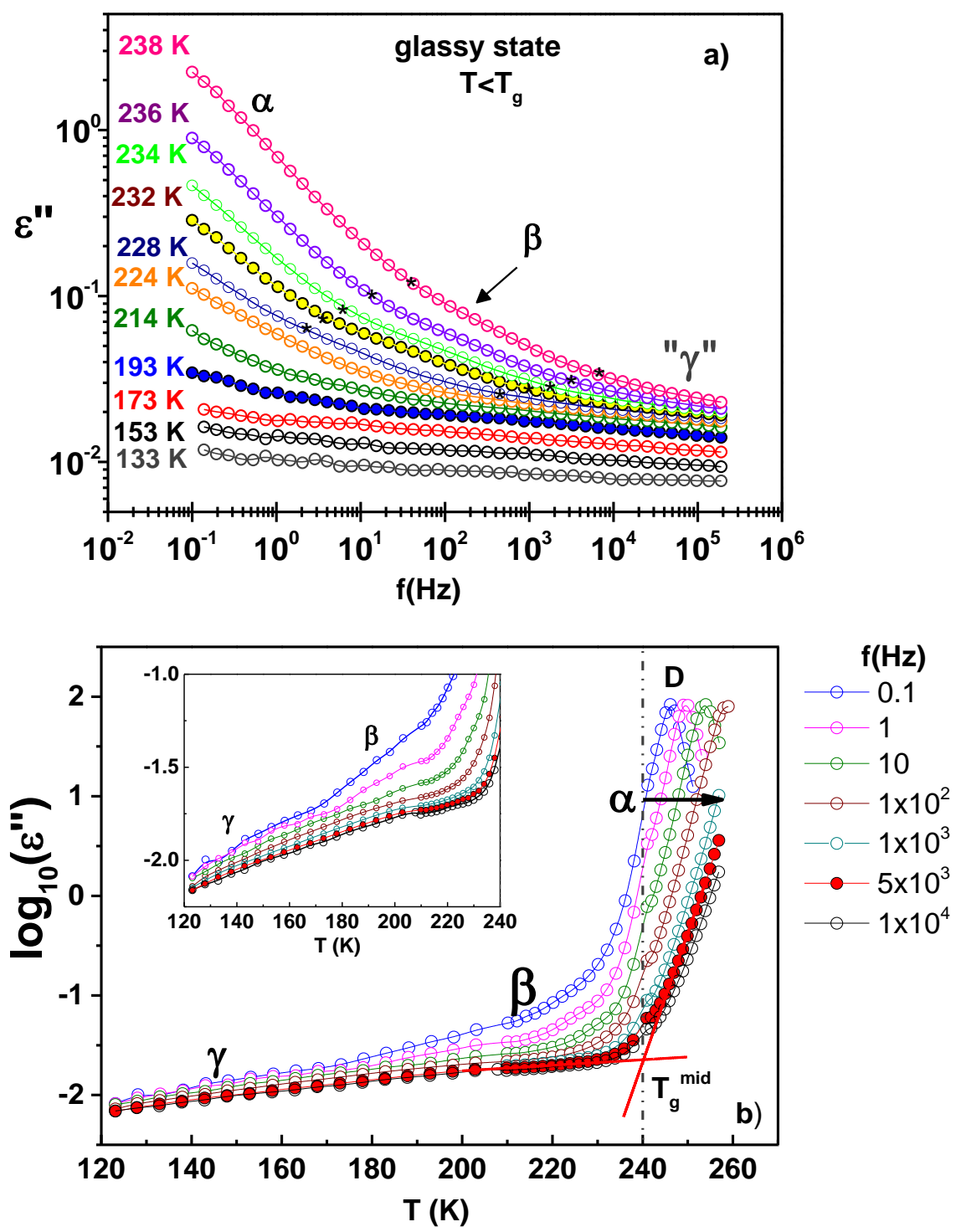

Figure 6: a) Frequency dependence of dielectric loss, $\varepsilon^{\prime \prime}$ of glassy Nac-MBA (ee $=50 \%$ ) at the indicated temperatures. Lines connect data points. b) Temperature dependence of $\log \left(\varepsilon^{\prime \prime}\right)$ at indicated frequencies (data taken from isothermal data). The insert shows a close up for $\mathrm{T}<\mathrm{T}_{\mathrm{g}}{ }^{\text {mid }}=240 \mathrm{~K}$.

Figure $7 a)$ and $7 b$ ) show illustrative dielectric spectra ( $\varepsilon^{\prime}$ and $\varepsilon^{\prime \prime}$ respectively) as a function of the available frequency range in the glassy state near below $T_{g}$ and in the supercooled liquid state. The slower D-relaxation visible as a step in $\varepsilon^{\prime}$ or as a very intense dielectric response $\varepsilon^{\prime \prime}$ (almost $90 \%$ of the dielectric strength) arises at temperatures above $246 \mathrm{~K}$. At T $>253 \mathrm{~K}$ dc conductivity (not shown for clarity reasons) rises at the lower frequency side of the D-process. In Fig $7 b$ ) it is shown that for $\mathrm{T}=259 \mathrm{~K}$ a Debye curve (solid red line) matches only the low 
frequency side $\mathrm{f}<10^{3} \mathrm{~Hz}$ of the $\varepsilon^{\prime \prime}(f)$ spectrum. The faster relaxation previously identified as the $\alpha$-relaxation is somewhat obscured under the high-frequency flank of the large amplitude D-process. In order to improve the frequency resolution of the overlapping dielectric loss peaks, the derivative method developed by Wübbenhorst and van Turnhout ${ }^{81}$ is applied:

$$
\varepsilon_{d e r}^{\prime \prime}=-\frac{\pi}{2} \frac{\partial \varepsilon^{\prime}(f)}{\partial \ln (2 \pi f)}
$$

This method has the advantage to considerably improve the resolution of overlapping peaks and also suppress Ohmic conduction because in that case $\varepsilon^{\prime}$ is independent of frequency. ${ }^{81}$ Figure 7c) shows that the $\alpha$-relaxation appears much more clearly from the derivative expression at intermediate frequency range between the slower D-process and the faster $\beta$ process. The conduction free dielectric loss $\varepsilon_{d e r}^{\prime \prime}$ data was analyzed with a sum of derivative $\mathrm{HN}$-functions in order to obtain the relaxation times corresponding to the frequency of maximal dielectric loss $\left(f_{\max }\right)$ for the $\mathrm{D}$ - and $\alpha$-relaxations. The calculated parameters were then used as starting values for a fitting procedure resulting in a reliable description of the raw data using a sum of Debye $\left(\alpha_{H N}=1, \beta_{H N}=1\right)$ and Cole-Cole $\left(\beta_{H N}=1\right)$ model functions (equation 3). For high temperatures, a dc conductivity contribution was also added to describe the raw dielectric loss data at the lower frequency side of the D-process.

Illustrative example of the fitting procedure used for a semi-quantitative analysis of the current data is given in figure 8 for $T=259 \mathrm{~K}$. The D-process for $\mathrm{T}>246 \mathrm{~K}$ is well described by a Debye model function ( $\alpha_{H N}=1, \beta_{H N}=1$ ) corresponding to a single exponential behavior in the time domain. Concerning the structural $\alpha$-relaxation $(T>241 \mathrm{~K}$ ) the parameter that quantifies the symmetric broadening remains constant in the supercooled liquid domain $\left(\alpha_{H N}=0.59 \pm 0.01\right)$. Normally, the fitting procedure permits to derive information about the shape parameters, the dielectric strength and the relaxation time of the different processes. In the present case such fitting procedure is somehow challenging and daring regarding poorly resolved or overlapping relaxations phenomena, and low magnitude processes. The uncertainties of the width parameters (and dielectric strengths) and their temperature dependencies obtained from the fits, especially for the $\beta$ and $\gamma$ relaxations, are rather large and must be taken indicative (see Supplementary materials). The temperature dependence of the relaxations times are now presented and discussed. 

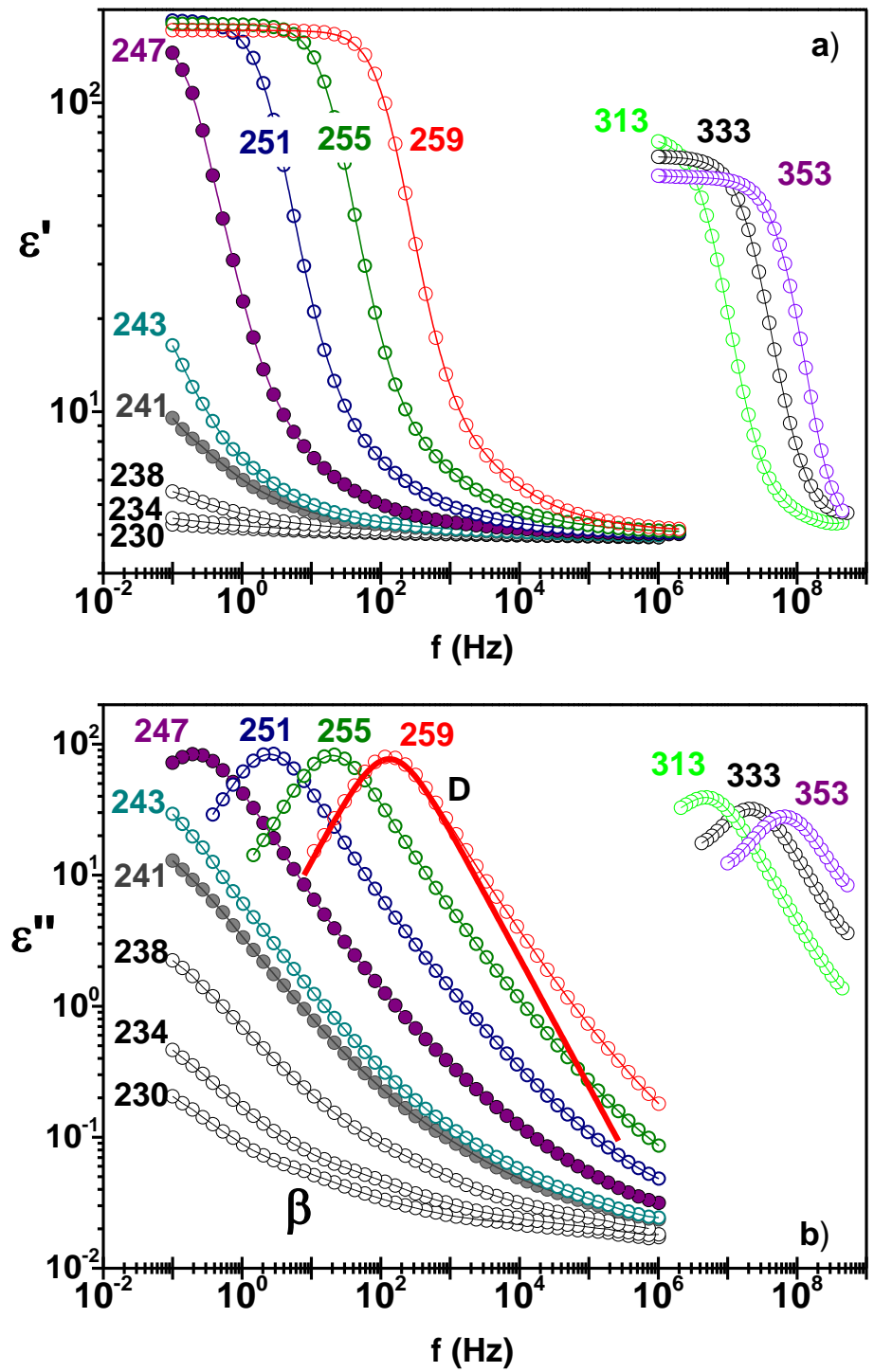


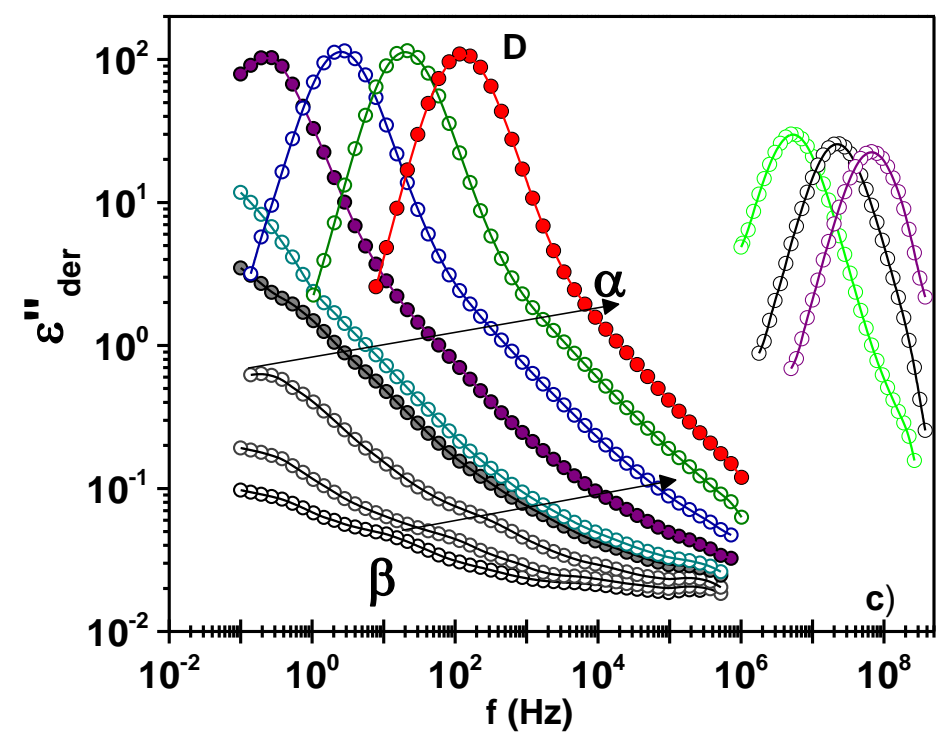

Figure 7: Frequency dependence of a) the real permittivity $\varepsilon^{\prime}$ b) dielectric loss and c) conduction free dielectric loss $\varepsilon_{d e r}^{\prime \prime}$, in the available frequency region [0.1Hz-1GHz] for the indicated temperatures given in Kelvin. Lines connect data points. In panel b) conductivity tail observed at lower frequencies than D-relaxation is omitted; Red solid line is the result of fitting a Debye function to the dielectric loss spectra at $259 \mathrm{~K}$.

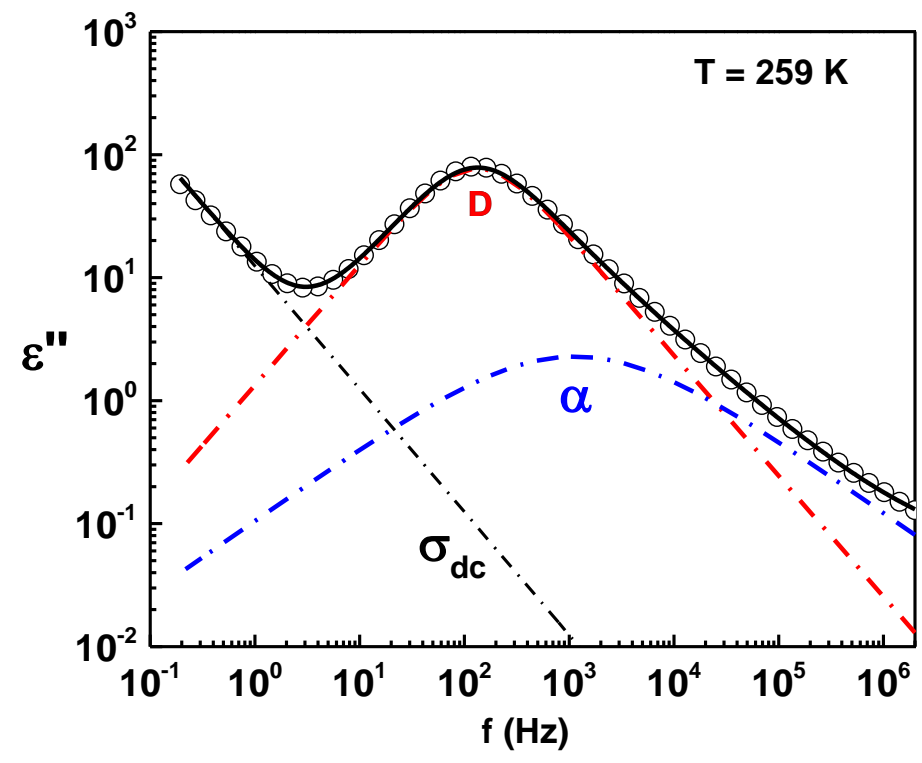

Figure 8: Fitting procedure of the dielectric loss peak in the supercooled liquid at 259 K.The dash-dot lines represent the individual fitting functions (Cole-Cole for the $\alpha$-relaxation and Debye for the D-process). Solid lines are the total fit functions (see text for details). 


\subsubsection{Relaxation map}

The temperature dependence of the relaxation time of all the four detected processes (Debye, $\alpha, \beta$ and $\gamma)$ is summed up in relaxation map $(-\log \tau$ vs $1 / T)$ shown in Figure 9. For sake of simplicity and clarity, the Arrhenius plot will be analyzed from the glassy state towards the supercooled liquid. The evaluated parameters from the applied model functions (Arrhenius and VFTH) used to describe the temperature dependence of the relaxation times of the various processes are summarized in table 1.
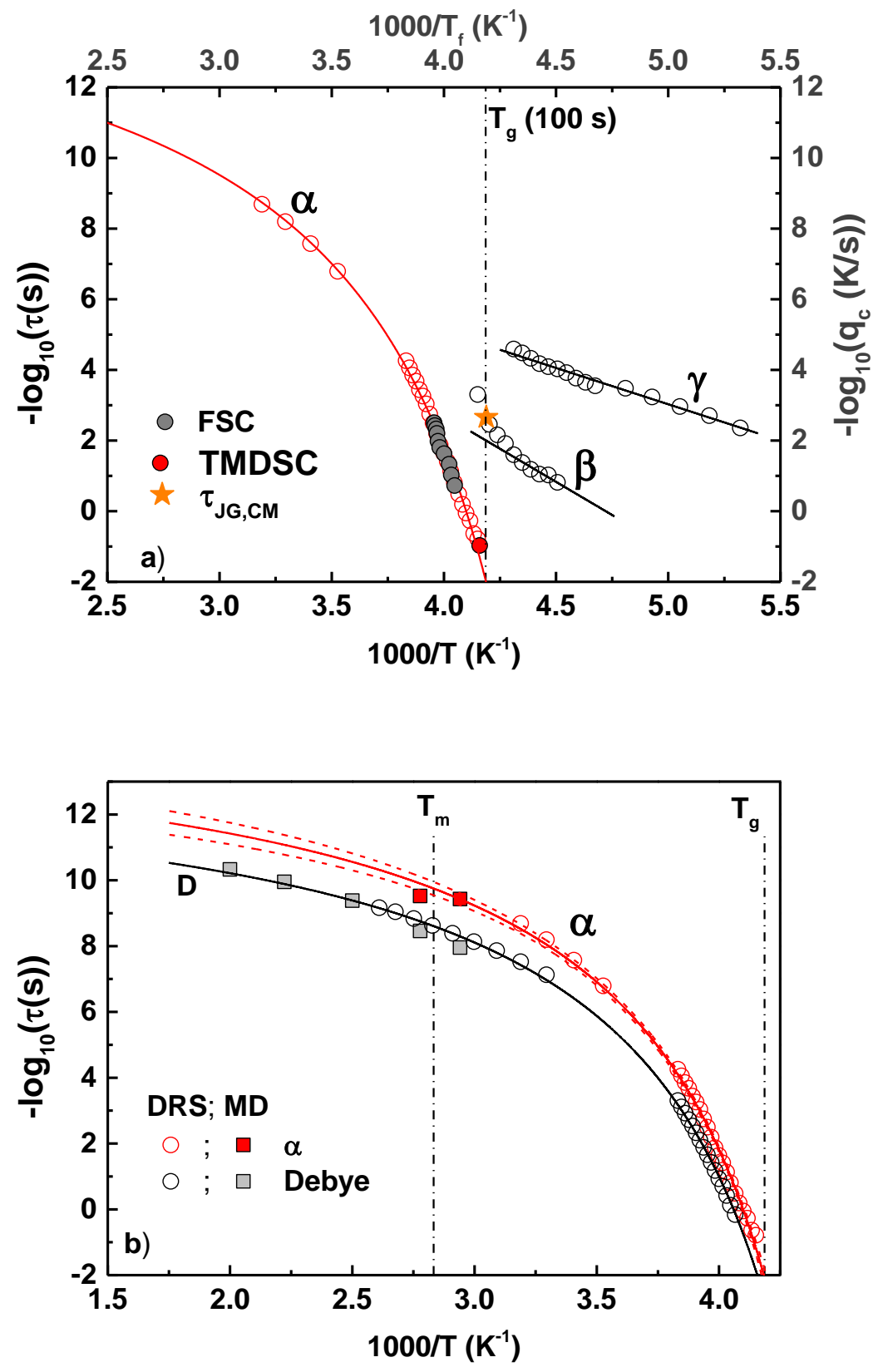

Figure 9: a) Relaxation map $(-\log \tau$ vs $1000 / T)$ for the $\alpha-, \beta$ - and $\gamma$-relaxations of Nac-MBA probed by DRS (vertical left-hand axis). The red filled circle corresponds to the kinetic glass 
transition temperature $\left(\mathrm{T}_{\mathrm{g}}{ }^{\mathrm{mid}}=240.4 \mathrm{~K} ; \tau=9.5 \mathrm{~s}\right)$. The orange star is the JG relaxation time calculated at $T_{g}$ using the $\mathrm{CM}$ (eq. (10)). Cooling rate (- $\left.\log q_{c}\right)$ as a function of the inverse of the fictive temperature $\left(T_{f}\right)$ determined from FSC (filled grey circles) is shown in the vertical right-hand axis. b) Relaxation map for the $\alpha$ - and Debye-like relaxations detected in the supercooled (above Tg) and liquid (above Tm) from DRS (open circles) and MD simulations (filled squares). Solid lines are fits of the Arrhenius and VFTH equation to the corresponding data. Dashed lines in panel b) are the $95 \%$ confidence limits of VFTH fit to the $\alpha$-relaxation.

Table 1: Fit parameters of the Arrhenius and VFTH functions used to describe the temperature evolution of the relaxation time of $\gamma$ - and $\beta$-secondary relaxations and $\alpha$ - and D-processes respectively.

$$
\begin{array}{ccc}
T<T_{g} & E_{a}\left(k J . m^{-1}\right) & \tau_{\infty}(\mathrm{s})
\end{array}
$$

\begin{tabular}{cccc}
\hline $\boldsymbol{\gamma}$ - process & $39.4 \pm 1.5$ & \multicolumn{2}{c}{$(6.6 \pm 4.5) \times 10^{-14}$} \\
$\boldsymbol{\beta}$-process & $71.8 \pm 8.0$ & $(5.37 \pm 5.36) \times 10^{-17}$ \\
\hline \hline $\boldsymbol{T}>\boldsymbol{T}_{\boldsymbol{g}}$ & B (K) & $\tau_{\infty}(\mathrm{s})$ & $T_{0}(\mathrm{~K})$ \\
\hline $\boldsymbol{\alpha}$-process VFTH (DRS) & $1310 \pm 90$ & $(1.3 \pm 0.5) \times 10^{-14}$ & $203 \pm 2$ \\
VFTH (DRS + MD) & $1123 \pm 66$ & $(8 \pm 2) \times 10^{-14}$ & $207 \pm 2$ \\
\hline D- process VFTH & $1024 \pm 38$ & $(2.0 \pm 0.2) \times 10^{-12}$ & $209 \pm 1$
\end{tabular}

- Molecular mobility below $T_{\mathrm{g}}: \boldsymbol{\gamma}$ - and $\boldsymbol{\beta}$ - relaxations

As generally observed for localized, non-cooperative motions observed in the glassy state, the relaxation time of both $\gamma$ - and $\beta$ - processes follows a linear temperature dependence of $\log \tau$ vs $1000 / T$ well described by the Arrhenius relation:

$$
\tau(T)=\tau_{\infty} \exp \left(\frac{E_{a}}{R T}\right)
$$

where $\tau_{\infty}$ is the pre exponential factor, $E_{a}$ the activation barrier energy and $R$ the ideal gas constant. Given the value of the activation barrier energy of the $\gamma$-process $\left(E_{a}^{\gamma} \sim 39 \mathrm{~kJ} / \mathrm{mol}\right)$ it is reasonable to suggest that this secondary relaxation may originate from intramolecular, small angular fluctuations of polar side groups of Nac-MBA molecules that could persist deep in the glassy state. Nevertheless, it should also mentioned that small amount of impurities can also be at the origin of such manifestation. ${ }^{82}$ 
The higher activation energy found for the $\beta$-process $\left(E_{a}^{\beta} \sim 72 \mathrm{~kJ} / \mathrm{mol}\right)$ can be compatible with a complete rotation of the whole molecule as suggested for the $\beta$ Johari-Goldstein $\left(\beta_{J G}\right)$ process when analyzed in the framework of the Coupling Model (CM)..$^{83}$

The temperature evolution of $\tau_{\beta}$ strongly deviates from the Arrhenius behavior at the vicinity of $T_{g}$. This peculiar change of $\tau_{\beta}(T)$ has been highlighted for many glass formers, ${ }^{12,78,84,85}$ and it is recognized as a manifestation of the $\beta_{J G}$-process. According to the $\mathrm{CM}$, the $\beta_{J G}$-process is considered as located near an independent or primitive motion (that implies the rotation of the whole molecule) precursor of the structural $\alpha$-relaxation. The relaxation time of the $\beta_{J G}$ process is approximatively the same as that of the characteristic time of the primitive motion $\left(\tau_{0}\right)$ and correlated to $\tau_{\alpha}$ through the following equation:

$$
\tau_{J G}(T) \approx \tau_{0}(T)=t_{c}^{n}\left[\tau_{\alpha}(T)\right]^{1-n}
$$

where $n$ is the coupling parameter $\left(n=1-\beta_{K W W}\right)$ and $t_{c}$ is the time characterizing the crossover from independent to cooperative fluctuations proven to be close to $t_{c}=$ $2 \times 10^{-12} s$ for molecular glass formers. ${ }^{86}$ From the experimental data it is very difficult to estimate a $\beta_{K W W}$ value due to the poor frequency resolution of the $\alpha$-relaxation in the dielectric loss spectrum. Though, a value $\beta_{K W W}=0.66$ can be estimated from the obtained $\mathrm{HN}$ shape parameters of the structural $\alpha$-relaxation ( $\alpha_{H N}=0.59$ and $\beta_{H N}=1$ ) using the empirical relation proposed by Alegria and coworkers: ${ }^{87}$

$$
\beta_{K W W} \approx\left(\alpha_{H N} \beta_{H N}\right)^{\frac{1}{1.23}}
$$

Taking into account the $\beta_{K W W}$ value in the $\mathrm{CM}$ relation and $\tau_{\alpha}=100 \mathrm{~s}$ at $T_{g}$, a value of $\tau_{J G}\left(T_{g}\right)=2.22 \times 10^{-3} \mathrm{~s}$ is obtained. This value is in good agreement with the relaxation time derived from the analysis of the dielectric loss data as shown in figure 9a). We must note that the most obvious manifestation of this secondary relaxation (a weak shoulder in the dielectric loss peak) takes place in a very narrow temperature range (almost $15 \mathrm{~K}$ ). These difficulties increase the uncertainties for a direct confirmation of the $\beta_{J G}$ nature of this slower secondary process. Therefore, empirical approaches such as the proposal of Ngai and Capacciolli ${ }^{88}$ can be applied:

$$
\frac{E_{a}}{R T_{g}}=2.303\left(2-13.7 n-\log \tau_{\infty}\right)
$$

In the present case, the ratio $\frac{E_{a}}{R T_{g}} \sim 31.7$ is approximately identical to the second term of the equation (12), $2.303\left(2-13.7 n-\log \tau_{\infty}\right) \sim 29.8$. The apparent correlation of $\tau_{\beta}$ with $\tau_{\alpha}$, its 
change at $T_{g}$ and the relationship of the activation energy of the $\beta$-process in the glassy state with $T_{g}$, suggest that the detected $\beta$-relaxation in Nac-MBA is a genuine Johari-Goldstein $\beta$ process.

\section{- Molecular mobility above $T_{g}$ : $\alpha$-relaxation and Debye-like process}

Figure 9a) includes data obtained from TMDSC (red full circle) and FSC (gray full circles) techniques allowing comparison with the dielectric $\alpha$-relaxation time $\tau_{\alpha}$ (red open circles). For the FSC data, a vertical shift factor (after the FKR relation (eq. 5), $\log C=0.67 \pm 0.12$ or $C=$ $(4.7 \pm 1.3) \mathrm{K}$, is necessary in order to superimpose the temperature dependence of the cooling rate $\left(-\log \left(q_{c}\right)\right.$ vs. $\left.1 / T_{f}\right)$ with the temperature behavior of the $\alpha$-relaxation time $\left(-\log \left(\tau_{\alpha}\right)\right.$ vs. $1 / T$ ). For the temperature range common to calorimetric and dielectric technique's ([240 K; $253 \mathrm{~K}$ ] - the TMDSC raw point is also included), a strong similarity of the slope of the two set of data is clearly evidenced which without a doubt confirms the evaluation of $\tau_{\alpha}$ using the fitting procedure described previously. The nonlinearity of the temperature dependence (or super-Arrhenius behavior) of the $\alpha$-relaxation time is often described by the empirical VogelFulcher-Tamman-Hesse (VFTH) equation given by: ${ }^{89-91}$

$$
\tau(T)=\tau_{\infty} \exp \left(\frac{B}{T-T_{0}}\right)
$$

where $\tau_{\infty}$ is the value of the relaxation in the high temperature limit, $B$ is an empirical parameter representative of the material that accounts for the deviation of linearity and $T_{0}$ is the so-called Vogel temperature or ideal glass transition temperature ( $0 K<T_{0}<T_{g}$ ). In the present case of Nac-MBA, the experimental data alone (Figure 9a)) or when combined with MD simulations (Figure 9b)) are fairly well described by a single VFTH function (see table 1) with physically acceptable values of the pre-exponential factor corresponding to phonon lifetime $\left(\tau_{\infty} \sim 10^{-14}-10^{-13} \mathrm{~s}\right)$. Note that the VFTH fit allows one to estimate, for $\tau_{\alpha}=9.5 \mathrm{~s}$, a temperature $\mathrm{T}_{\alpha}(9.5 \mathrm{~s})=241.3 \pm 0.3 \mathrm{~K}$ which agrees well with $\mathrm{T}_{\mathrm{g}}{ }^{\mathrm{mid}}=240.4 \pm 0.5 \mathrm{~K}$ obtained from the specific heat capacity curve (TMDSC) at the equivalent frequency $(\sim 0.02 \mathrm{~Hz}$ and heating rate $0.5 \mathrm{~K} / \mathrm{min})$. The dielectric glass transition temperature $\mathrm{T}_{\mathrm{g}}{ }^{\mathrm{DRS}}(100 \mathrm{~s})=238.8 \pm 0.4$ $\mathrm{K}$ is obtained by extrapolating the VFTH fit to the relaxation time $\tau=100 \mathrm{~s}$. 
Usually, the temperature evolution of the $\alpha$-relaxation time of all glass formers do not express the same deviation from an Arrhenius behavior near $T_{g}$. The quantification of this deviation is at the origin of the distinction between fragile and strong systems. This deviation near $\mathrm{T}_{\mathrm{g}}$ can be quantified by the so called fragility (or steepness) index $m$ defined as follow: ${ }^{92}$

$$
m=\left[\frac{d \log \tau_{\alpha}}{d\left(T / T_{g}\right)}\right]_{T=T_{g}}
$$

A glass former is considered as strong when $m \leq 30$ and fragile when $m \geq 100$. For the particular case where $30<m<100$ the liquid can be considered as an intermediate glass former. Combining Eq. 13 and Eq. 14, $m$ can be expressed as:

$$
m=\frac{B T_{g}}{\ln 10\left(T_{g}-T_{0}\right)^{2}}
$$

Considering the values of $B$ and $T_{0}$ reported in table 1 (for DRS and DRS+MD data set) an average value of $m=111 \pm 7$ is obtained, allowing one to classify Nac-MBA as a fragile glass former. The secondary amide Nac-MBA here studied appears a much more fragile system than $\mathrm{N}$-ethylacetamide and its mixtures with $\mathrm{N}$-methylformamide for which a fragility index $\mathrm{m}=65$ has been reported. ${ }^{31}$

As depicted in Figure 9b), where DRS and MD data obtained in the supercooled liquid and liquid state are combined, the temperature dependence of the D-process relaxation times, $\tau_{D}$ also be described by a VFTH equation. The obtained values of the B parameter and of the Vogel temperature $T_{0}$ much resemble the ones of the VFTH used to describe the temperature dependence of $\tau_{\alpha}$ (table 1). These similarities between both relaxations could possibly mean that D-process may be controlled or governed by $\alpha$-relaxation. ${ }^{12}$ The non-Arrhenius temperature dependence of $\tau_{\alpha}$ is highly correlated with a non-Debye relaxation dynamics. However the Debye-like process is not liable to any dispersion of the relaxation time and consequently cannot originates from the same microscopic phenomena as the $\alpha$-process. ${ }^{31,93}$ The ratio of the two times scales $\tau_{D} / \tau_{\alpha}=8$ at $\mathrm{T}\left(\tau_{D}=100 \mathrm{~s}\right)=241 \mathrm{~K}\left(\sim 2 \mathrm{~K}\right.$ above $\left.\mathrm{T}_{\mathrm{g}}{ }^{\mathrm{DRS}}\right)$; near the melting temperature $T_{\mathrm{m}}\left(\sim 30\right.$ at $\mathrm{T}=340 \mathrm{~K}$ from MD) and for $T \rightarrow \infty, \tau_{D} / \tau_{\alpha}=25$. The obtained ratio is in agreement with those observed in $\mathrm{N}$-ethylacetamide and its mixture with $\mathrm{N}$-methylformamide, ${ }^{31,32}$ and some monohydroxy alcohols. ${ }^{36,41,94}$

The microscopic origin of D-process and its implications in the dielectric response of the investigated Nac-MBA system (ee $=50 \%$ ) are probed by MD simulations and presented in the following section. 


\subsection{MD simulations investigations}

\subsubsection{Static dipolar properties}

The individual dipole moment of a given molecule at time $t$ is calculated from the classical expression $\vec{\mu}(t)=\sum_{\alpha=1}^{\mathrm{N}_{\mathrm{a}}} q_{\alpha} \vec{r}_{\alpha}(t)$ where $q_{\alpha}$ and $\vec{r}_{\alpha}(t)$ are respectively the fixed charge localized on atom $\alpha$ and its position at time $t$, and $\mathrm{N}_{\mathrm{a}}=25$ is the number of atoms in the considered Nac-MBA molecules $\mathrm{C}_{10} \mathrm{H}_{13} \mathrm{NO}$. Fixed charges are directly taken from the ab-initio calculation (see section 2.5 MD simulations details). In the present investigation, the average dipole moment $\langle\mu>$ of Nac-MBA molecules computed from MD is found to be about 3.93 +/- 0.01 Debye in the liquid state, and does not significantly vary with temperature. The symbol < > indicates an average on both time and molecules. It could be noted that this value is close to the value 3.74 Debye directly obtained from the ab-initio calculations of a single molecule in the gas phase (see section 2.5).

Dipolar correlations are well described by the so-called Kirkwood correlation factor $\mathrm{G}_{\mathrm{K}}$ given by the following equation ${ }^{95}$

$$
\mathrm{G}_{\mathrm{K}}=\mathbf{1}+(\mathbf{N}-\mathbf{1})\left\langle\vec{\mu}_{i} \cdot \vec{\mu}_{j}\right\rangle /\left\langle\mu^{2}\right\rangle
$$

where $\vec{\mu}_{i}$ is the dipole moment of the molecule $i,\left\langle\mu^{2}\right\rangle$ the average dipole moment, and $\left\langle\vec{\mu}_{i} \cdot \vec{\mu}_{j}\right\rangle$ indicates an average over distinct pairs of molecules $(i \neq j)$. The Kirkwood correlation factor accounts for the orientational correlation of neighboring dipoles. Parallel and antiparallel dipoles orientation lead to $\mathrm{G}_{\mathrm{K}}>1$ and $\mathrm{G}_{\mathrm{K}}<1$, respectively. If orientations of individual dipole are completely random, one finds $\mathrm{G}_{\mathrm{K}}=1$. The Kirkwood correlation factor $\mathrm{G}_{\mathrm{K}}$ was calculated at different temperatures from Eq. 16 and it is shown in figure 10. The Kirkwood correlation factor monotonically increases from about 1.6 at $\mathrm{T}=500 \mathrm{~K}$ to about 2.6 at $\mathrm{T}=340 \mathrm{~K}$. 


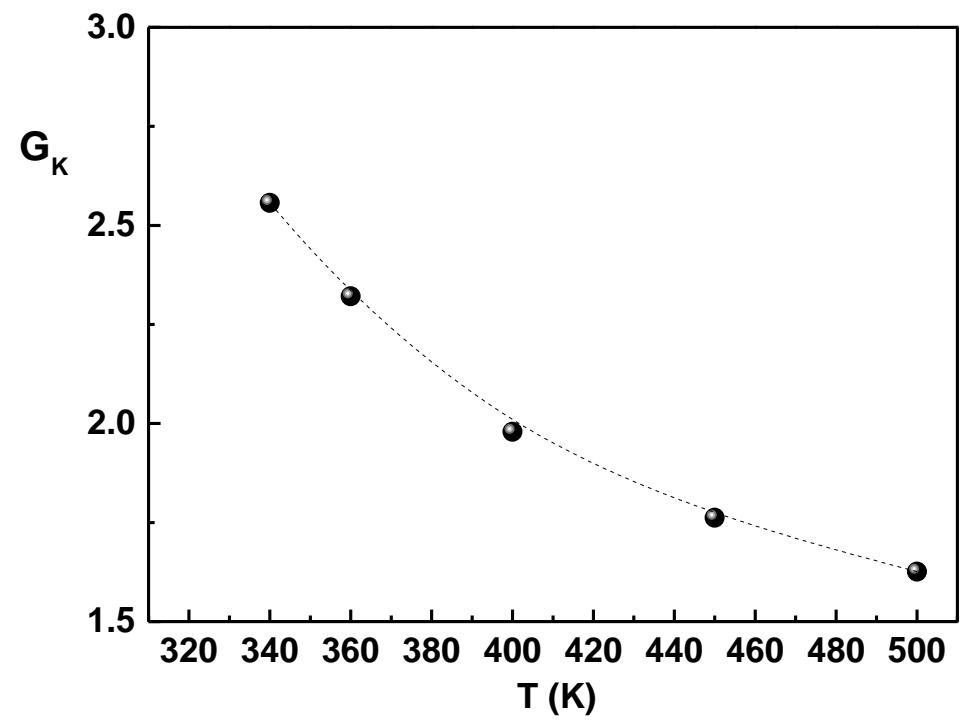

Figure 10: Temperature evolution of the Kirkwood correlation factor $G_{K}$ of $N a c-M B A$ (ee=50\%) determined from MD simulations.

A Kirkwood factor $G_{K}$ greater than 1 is often found in monohydroxy alcohols $25,43,96,97$ and secondary amides ${ }^{31,32}$ for which values may range from 3 up to 4 . It is interpreted as the capability of these liquids to form HB associating structures as linear chains showing HB aggregates with parallel dipoles orientation. This type of HB associations has been often detected from the present MD investigations from snapshots of instantaneous configuration of the system, and will be discussed below. An example of an $n=6$ Nac-MBA molecules HB aggregate is displayed in figure 11. It should be also noted that the $G_{K}$ values increase with decreasing temperature suggesting an increasingly parallel correlation of dipoles which can be seen as a precursor of the crystalline structure that may ultimately formed as observed many times in HB molecular liquids. ${ }^{12,98}$ The crystalline structure of Nac-MBA suggests that the strongest intermolecular link is a HB between $N-H \cdots O$ atoms with $d(N-O)=2.87 \AA$, $d(H \cdots O)=2.10 \AA ; \quad((N-H \cdots O)=171.8 \mathrm{deg}$. The structure reveals infinite syndiotactic linear chains of $\mathrm{H}$-bonded molecules alternately parallel to $\langle 110\rangle$ and $\langle 1-10\rangle$ in successive (004) slices. More details about the crystalline structure can be found in the work of Druot et al. ${ }^{47}$

In other words, the latter hypothesis is consistent with the main feature of the crystalline structure (being the steric accessibility of the NH group leading to the presence of infinite linear $\mathrm{H}$-bond chains of molecules between $N-H \cdots O$ atoms). ${ }^{49}$ Though in the liquid or 
supercooled liquid state this infinite intermolecular associations cannot be envisaged (lack of long range order), it is very likely that as temperature fluctuation increases the number of molecules implicated in these associations diminishes. The high dielectric intensity values may emanate from the linear nature of the $\mathrm{H}$-bonding chains and linked to the number of molecules involved in these intermolecular associations.

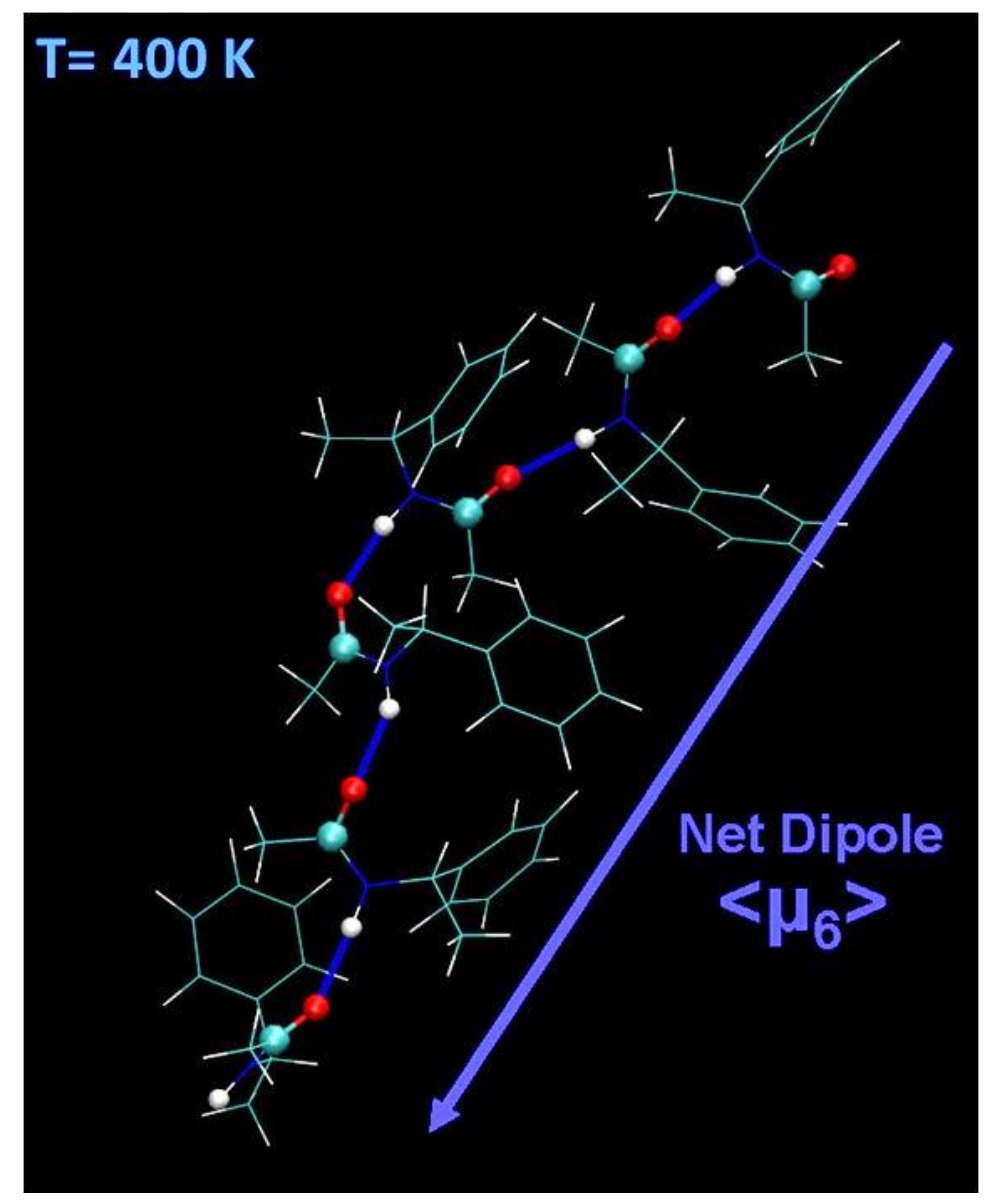

Figure 11: Snapshot extracted from an MD simulation run at $\mathrm{T}=400 \mathrm{~K}$ showing an $\mathrm{HB}$ aggregate composed of $n=6$ Nac-MBA molecules. This HB aggregate possesses a linear shape and the net dipole $\left\langle\mu_{6}\right\rangle$ of this aggregate is also schematically represented. Intermolecular HB are displayed as bold line.

\subsubsection{Dynamical dipolar properties}

Molecular mobility can be probed from the time dependence of the total electric dipole moment $\vec{M}(t)$ at time t. ${ }^{99}$ The total electric dipole moment is defined as $\vec{M}(t)=\sum_{i=1}^{N} \vec{\mu}_{i}(t)$ where $\vec{\mu}_{i}(t)$ is the individual dipole moment of the molecule $\mathrm{i}$ at time $t$ and $\mathrm{N}=64$ the total number of molecules. In a first approximation, the complex frequency dependent permittivity $\varepsilon^{*}(f)=\varepsilon^{\prime}(f)-i \cdot \varepsilon^{\prime \prime}(f)$ measured experimentally by DRS is actually proportional to the 
Fourier transform of the time-dependent correlation function $\Phi_{M}(t)=\langle\vec{M}(t) \cdot \vec{M}(0)\rangle$ of the total dipole moment. ${ }^{11}$ This function allows probing the collective dynamics of Nac-MBA dipoles and has been calculated at different temperatures (see figure 12). The long time behavior of the $\Phi_{M}(t)$ functions is dominated by a single exponential decay which can be well fitted with $\sim \exp \left[-t / \tau_{D}^{(M D)}\right]$, where $\tau_{D}^{(M D)}$ is the characteristic time associated with this long time decay. This behavior is well in line with the Debye like process observed by DRS. The single exponential law allowed us to reproduce the evolution of $\Phi_{M}(t)$ almost over the whole investigated time domain except at relatively short times $(10<\mathrm{t}<100 \mathrm{ps})$ where an additional fast relaxational process is present (see figure 12). This fast process possesses a very low intensity and a reliable characterization is difficult from the present MD data. It has thus just been taken into account considering an additional law $\sim \exp \left[-\left(t / \tau_{\text {fast }}^{(M D)}\right)^{\beta_{\text {fast }}^{(M D)}}\right]$ in order to reproduce the behavior of the $\Phi_{M}(t)$ function over the complete investigated time range. $\beta_{\text {fast }}^{(M D)} \approx 0.82$ is found to provide a reasonable adjustment in the fitting procedures.

The characteristic times $\tau_{D}^{(M D)}$ and $\tau_{\text {fast }}^{(M D)}$ obtained at different temperatures have been represented in Figure 9b) for comparison with the DRS experimental data. It clearly shows that the characteristic times determined from MD simulations are in very fair agreement respectively with the relaxation times of the $D$ - and $\alpha$ - processes obtained from DRS experiments. Both processes are separated by about one to 1.5 decades in time and closely follow each other's in the investigated temperature domain very much like the behavior observed in monohydroxy alcohols and secondary amides. 


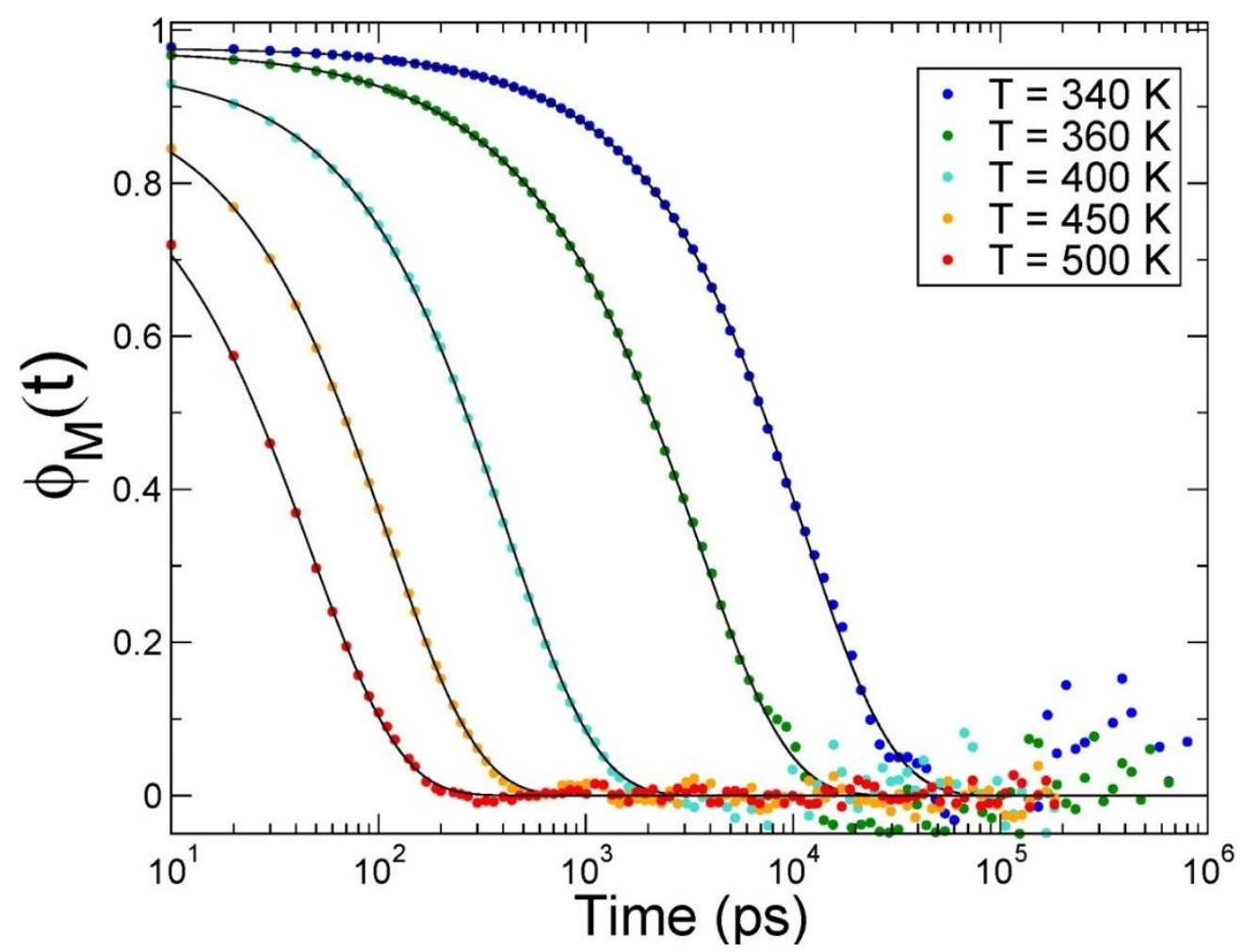

Figure 12: Time-dependent of the total dipole correlation function $\Phi_{M}(t)=\langle\vec{M}(t) \cdot \vec{M}(0)\rangle$ for Nac-MBA represented at different temperatures $\mathrm{T}=340,360,400,450$, and $500 \mathrm{~K}$. The solid lines indicate a fit with the function $\Phi_{M}(t)=A_{D}^{(M D)} \exp \left[-t / \tau_{D}^{(M D)}\right]+A_{\text {fast }}^{(M D)} \exp \left[-\left(t / \tau_{\text {fast }}^{(M D)}\right)^{\beta_{\text {fast }}^{(M D)}}\right]$ where the index “ $D$ " and "fast" refer to the long time single exponential decay (D-process) and to the fast relaxational process $(10<\mathrm{t}<100 \mathrm{ps})$ respectively (see text).

\subsubsection{Hydrogen bonding structures}

HB statistics allow determining the population of different $\mathrm{HB}$ associating structures and probing the structure of the investigated liquid with the aim to clarify experimental and numerical dipolar properties. The investigated molecule Nac-MBA possesses both a $-\mathrm{NH}$ and $\mathrm{C}=\mathrm{O}$ group from which they may form intermolecular HBs (see figure 11). In the present study, two Nac-MBA molecules are considered to be $\mathrm{H}$-bonded if $\mathrm{i}$ ) the nitrogen-oxygen distance is less than $3.4 \AA$ and ii) the $(\mathrm{O} \cdots \mathrm{H}-\mathrm{N})$ angle is larger than $150 \mathrm{deg}$. This criterion is classically used in MD simulations and allows including more deformed and weaker HBs in statistics. ${ }^{100}$ The fraction $P_{n}$ of HB aggregates composed of $n$ Nac-MBA molecules detected on average during the MD simulation is displayed in figure 13 at the highest $T=500 \mathrm{~K}$ and the lowest $\mathrm{T}=$ $340 \mathrm{~K}$ investigated temperatures. A monotonic trend of the $\mathrm{HB}$ statistics is found from $500 \mathrm{~K}$ 
down to $340 \mathrm{~K}$ and data obtained at 450, 400 and $360 \mathrm{~K}$ are not shown for clarity. Figure 13 clearly shows that a large variety of HB aggregates are theoretically possible ranging from isolated molecule $(n=1)$ to very large aggregates made of $n=20$ molecules. Additional even larger associated structures could be also transiently observed but their fraction remains really very small at all investigated temperatures. As expected from results obtained on dipolar correlations in the present study, the dominant intermolecular HB association originates from the formation of linear chain. As an example, a snapshot of a $n=6 \mathrm{HB}$ aggregate is displayed in figure 11. Upon decreasing temperature, the fraction of isolated molecules $(n=1)$ and dimers $(n=2)$ progressively decreases. The population of trimers $(n=3)$ remains stable while the population of $n>3$ aggregates increases. At the lowest investigated temperatures $T=340 \mathrm{~K}$, associations as dimer $(n=2)$ and trimer $(n=3)$ are seen as the most probable structures. However, it may be speculated that the trend found in the investigated temperature range will continue somehow at lower temperatures at which larger structures $\mathrm{n}>3$ might become the most probable. It is confirmed by simply plotting the number of molecule $\mathrm{n}$ corresponding to the maximum of $P_{n}$ as a function of temperature (data not shown). This evolution shows a monotonic increase of $n$ upon decreasing temperature. Additional MD simulations are clearly required that unfortunately extends beyond the scope of this study due to the difficulty to equilibrate liquid states at temperatures below $\mathrm{T}=340 \mathrm{~K}$ from MD because of the slow dynamics of the dominant $D$ process (see below).

In addition to the population $P_{n}$ of a given HB aggregate of size $n$, the average total dipolar moment $\left\langle\mu_{n}\right\rangle$ for this aggregate may also be computed (see figure 13 ). The evolution of $\left\langle\mu_{n}\right\rangle$ as a function of $\mathrm{n}$ clearly shows that $\left\langle\mu_{n}\right\rangle$ increases with $\mathrm{n}$, which is well in line with the linear nature of the HB aggregates (see snapshot on figure 11) and the Kirkwood correlation factor $\mathrm{G}_{\mathrm{K}}>1$ (see figure 10). Cyclic aggregates would cause $\left\langle\mu_{n}>\right.$ to decrease or even to go to zero. Interestingly, it may be also mentioned that the evolution of $\left\langle\mu_{n}\right\rangle$ is quite temperature-independent. All $\left\langle\mu_{n}>\right.$ data at $\mathrm{T}=340$ and $500 \mathrm{~K}$ overlap onto the same curve as well as $\mathrm{T}=360,400$ and $450 \mathrm{~K}$ data (data not shown). It suggests that the same types of association may potentially form at all temperature whose the exact conformation - linear chain above all - does not change very much with temperature. From both $P_{n}$ and $\left\langle\mu_{n}\right\rangle$, it is also possible to calculate the product $P_{n} \cdot\left\langle\mu_{n}\right\rangle$ which provides an estimation of the "dipolar weight" of an aggregate made of $n$ molecules. At T $=340 \mathrm{~K}$, although the population 
of aggregates made of $n=4,5$ or 6 molecules is weaker than $n=2$ or 3 , they could contribute to a larger extend to the dipolar response since they possess a total higher dipole moment.

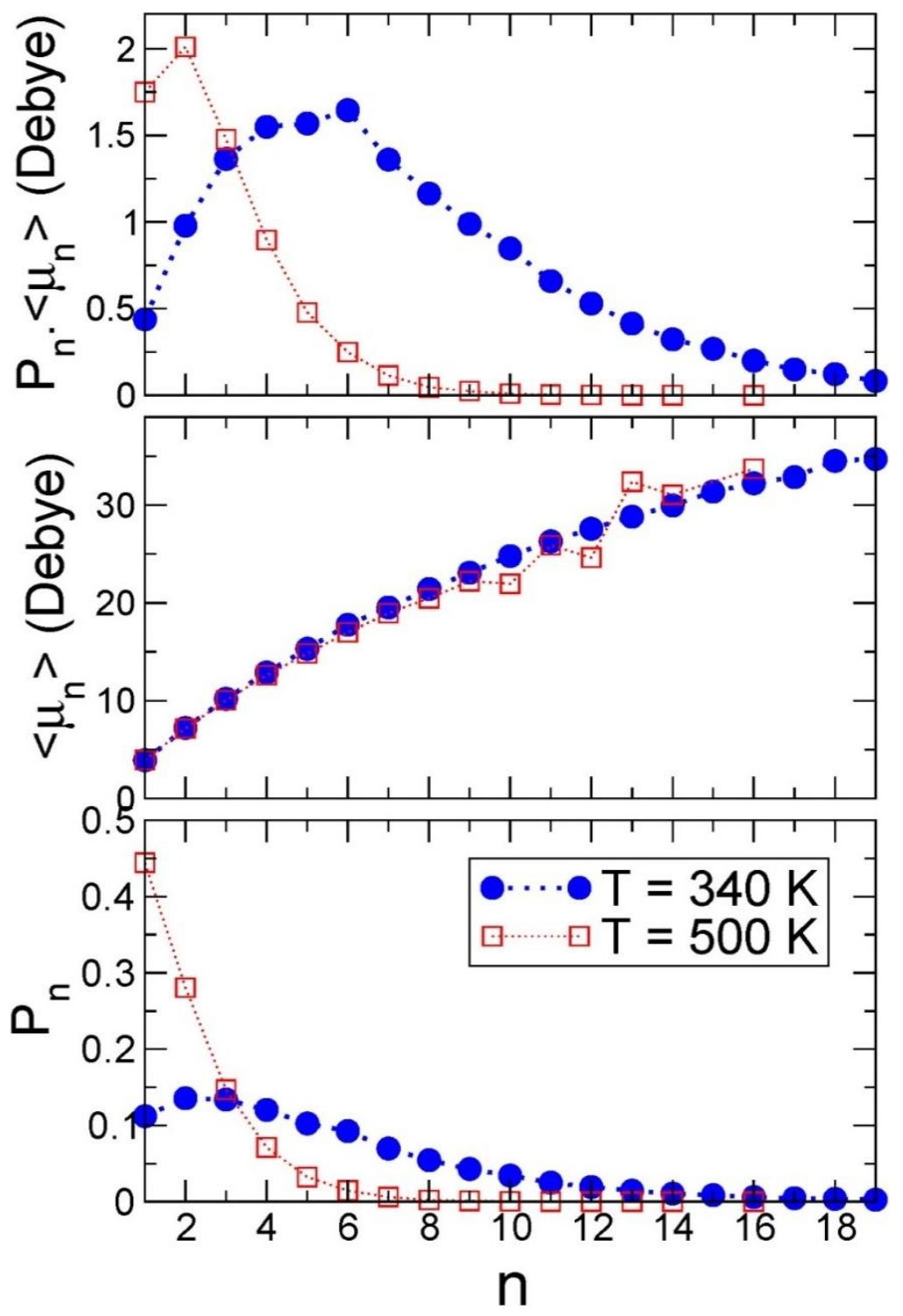

Figure 13: Evolution of the population $\boldsymbol{P}_{n}$ (bottom), the average net dipole $\left\langle\mu_{n}\right\rangle$ (middle) and the product $\boldsymbol{P}_{n} \cdot \boldsymbol{\mu}_{n}$ (top) for HB aggregates made of $\mathrm{n}$ Nac-MBA molecules at $\mathbf{T}=\mathbf{3 4 0}$ (circles) and $500 \mathrm{~K}$ (squares). 


\section{Conclusions}

Molecular mobility investigations in amorphous Nac-MBA system were achieved by combining experimental and simulation tools such as DRS, FSC and MD simulations covering over 12 decades in the frequency window. As many glass formers, Nac-MBA revealed a complex relaxational landscape. But interestingly a low frequency high intensity Debye-like process in the supercooled and liquid is observed by DRS and MD simulations, similarly to monohydroxy alcohols and secondary amides.

A secondary $\beta$-relaxation is detected in the glassy state and presents some characteristics (including a good agreement of its characteristic time at $\mathrm{T}_{\mathrm{g}}$ with that predicted from the $\mathrm{CM}$ model) that suggest that is a genuine Johari-Goldstein relaxation type.

Due to the high amplitude of the dominant D-relaxation, the visualization and localization of the $\alpha$-relaxation in the frequency dependence of dielectric loss data seems fastidious. Somehow, the derivative method applied on experimental raw $\varepsilon^{\prime}$ data permitted improving the frequency resolution of $\alpha$-process. The temperature dependence (fictive temperature) of the cooling rate performed by FSC mimics with an excellent agreement $\tau_{\alpha}(\mathrm{T})$ in the common probed temperature range. $\tau_{\alpha}(T)$ follows a single VFTH temperature evolution and the evaluated dielectric $\mathrm{T}_{\mathrm{g}}{ }^{\mathrm{DRS}}$ agrees well with the kinetic $\mathrm{Tg}^{\mathrm{mid}}$ from TMDSC. The deviation from the Arrhenius behavior at $T_{g}$ measured by the fragility index $(m=111 \pm 7)$ revealed that Nac-MBA can be classified as a fragile glass forming liquid.

The relaxation time of the highly intense monodispersive D-process that appears at lower frequencies in comparison with the structural relaxation is well describe by a VFTH law and the fit parameters show some similarities with that of $\alpha$-relaxation. This may be an indication that the dynamics of D-process is influenced by the $\alpha$-relaxation. The Debye-like process contributes almost for $90 \%$ in the global dielectric polarization and does not present calorimetric signature.

The Debye like process is clearly observed from MD simulations and its characteristic relaxation times $\tau_{D}$ are in very good agreement with high frequency DRS measurements confirming the validity of the calculations.

MD simulations also allowed to evidence in the liquid state the existence of HB aggregates of different size $n$ involving dimers, trimers, tetramers (e.g., $n=2, n=3, n=4$ ) and so on which can be mainly described as HB linear chains. As temperature decreases, longer $\mathrm{H}$-bonded chains 
become more probable. It is suggested that this trend could persist even in the supercooled liquid state and that HB linear chains of finite size could be thus seen as precursors of the future crystalline structure to grow composed of infinite HB chains.

$\mathrm{HB}$ linear chains possess parallel dipole orientation consistently with the Kirkwood correlation factor $G_{K}>1$ like the behavior reported for monohydroxy alcohols and secondary amides. Upon decreasing temperature, the Kirkwood correlation factor $G_{K}$ increases well in line with the formation of longer H-bonded linear chains. Effective "super-dipoles" are actually generated by these chains and contribute to the dielectric response observed experimentally. Although longer HB chains are less probable than shorter chains, they could contribute to a larger extend to the dipolar response since they possess a higher "super-dipole" moment. Interestingly, the value of the "super-dipole" of a given HB linear chain of size $n$ does not vary much with temperature implying that the "linearity" of these chains remains similar at all temperatures. This result nicely confirms that the hydrogen bond induces very strong directional intermolecular interactions which are absent in other non-associated liquids.

Globally, the results presented here for the secondary amide NAC-MBA, reinforce and confirmed the idea proposed in the literature for amides and monohydroxy alcohols that the high intense single exponential dielectric response originates from its rich microstructure due to hydrogen bonds. In the present study, only one excess enantiomeric ratio has been investigated ee $=50 \%$, it should be interesting to investigate other ratios to evaluate the impact of chirality on the Debye process which possibility could lead to additional insights on its still unclear origin.

\section{Acknowledgments}

The authors are grateful to FEDER and Region Normandie for the financial support of this work through FEDER MACHI project. This project has received funding from the Interreg 2 Seas program 2014-2020 co-funded by the European Regional Development Fund under subsidy contract 2S01-059_IMODE. The authors acknowledge the use of the facilities of the CRI (Villeneuve d'Ascq, France) where calculations were carried out.

\section{Conflicts of interest}

There are no conflicts to declare. 


\section{References}

1 C. A. Angell, Glass-Formers and Viscous Liquid Slowdown since David Turnbull: Enduring Puzzles and New Twists, MRS Bull., 2008, 33, 544-555.

2 P. G. Debenedetti, Metastable Liquids: Concepts and Principles, Princeton University Press, 1996.

3 R. Richert and A. Blumen, in Disorder Effects on Relaxational Processes, Springer, Berlin, Heidelberg, 1994, pp. 1-7.

4 M. D. Ediger, C. A. Angell and S. R. Nagel, Supercooled Liquids and Glasses, J. Phys. Chem., 1996, $100,13200-13212$.

5 C. A. Angell, K. L. Ngai, G. B. McKenna, P. F. McMillan and S. W. Martin, Relaxation in glassforming liquids and amorphous solids, J. Appl. Phys., 2000, 88, 3113-3157.

6 K. L. Ngai, Dynamic and thermodynamic properties of glass-forming substances, J. Non-Cryst. Solids, 2000, 275, 7-51.

7 E. Donth, The Glass Transition: Relaxation Dynamics in Liquids and Disordered Materials, Springer Science \& Business Media, 2013.

8 M. Descamps and J.-F. Willart, in Disordered Pharmaceutical Materials, ed. rc Descamps, WileyVCH Verlag GmbH \& Co. KGaA, 2016, pp. 1-56.

9 F. Stickel, E. W. Fischer and R. Richert, Dynamics of glass-forming liquids. II. Detailed comparison of dielectric relaxation, dc-conductivity, and viscosity data, J. Chem. Phys., 1996, 104, 2043-2055.

$10 \mathrm{~F}$. Kremer and A. Schönhals, in Broadband Dielectric Spectroscopy, Springer, Berlin, Heidelberg, 2003, pp. 99-129.

11 P. Lunkenheimer, U. Schneider, R. Brand and A. Loid, Glassy dynamics, Contemp. Phys., 2000, 41, $15-36$.

12 A. R. Brás, J. P. Noronha, A. M. M. Antunes, M. M. Cardoso, A. Schönhals, F. Affouard, M. Dionísio and N. T. Correia, Molecular Motions in Amorphous Ibuprofen As Studied by Broadband Dielectric Spectroscopy, J. Phys. Chem. B, 2008, 112, 11087-11099.

13 B. Schammé, M. Mignot, N. Couvrat, V. Tognetti, L. Joubert, V. Dupray, L. Delbreilh, E. Dargent and G. Coquerel, Molecular Relaxations in Supercooled Liquid and Glassy States of Amorphous Quinidine: Dielectric Spectroscopy and Density Functional Theory Approaches, J. Phys. Chem. B, 2016, 120, 7579-7592.

14 K. Grzybowska, K. Adrjanowicz and M. Paluch, in Disordered Pharmaceutical Materials, ed. rc Descamps, Wiley-VCH Verlag GmbH \& Co. KGaA, 2016, pp. 301-360.

15 B. Rijal, L. Delbreilh and A. Saiter, Dynamic Heterogeneity and Cooperative Length Scale at Dynamic Glass Transition in Glass Forming Liquids, Macromolecules, 2015, 48, 8219-8231.

16 G. Floudas, M. Paluch, A. Grzybowski and K. Ngai, Molecular Dynamics of Glass-Forming Systems: Effects of Pressure, Springer-Verlag, Berlin Heidelberg, 2011.

17 F. Kremer and A. Loidl, Eds., The Scaling of Relaxation Processes, Springer International Publishing, 2018.

$18 \mathrm{E}$. W. Fischer, E. Donth and W. Steffen, Temperature dependence of characteristic length for glass transition, Phys. Rev. Lett., 1992, 68, 2344-2346.

19 A. Saiter, L. Delbreilh, H. Couderc, K. Arabeche, A. Schönhals and J.-M. Saiter, Temperature dependence of the characteristic length scale for glassy dynamics: Combination of dielectric and specific heat spectroscopy, Phys. Rev. E, 2010, 81, 041805.

20 B. Rijal, J. A. Soto Puente, B. Atawa, L. Delbreilh, K. Fatyeyeva, A. Saiter and E. Dargent, Correlated and cooperative motions in segmental relaxation: Influence of constitutive unit weight and intermolecular interactions, Phys. Rev. E, 2016, 94, 062502.

$21 \mathrm{M}$. A. Floriano and C. A. Angell, On the relaxation between Debye and nonexponential relaxation in supercooled monohydric alcohols and water: A solution study, J. Chem. Phys., 1998, 91, 2537.

$22 \mathrm{U}$. Kaatze, Dielectric and structural relaxation in water and some monohydric alcohols, J. Chem. Phys., 2017, 147, 024502.

23 W. Dannhauser, Dielectric Study of Intermolecular Association in Isomeric Octyl Alcohols, J. Chem. Phys., 1968, 48, 1911-1917. 
24 C. Hansen, F. Stickel, T. Berger, R. Richert and E. W. Fischer, Dynamics of glass-forming liquids. III. Comparing the dielectric $\alpha$ - and $\beta$-relaxation of 1-propanol and o-terphenyl, J. Chem. Phys., 1997, 107, 1086-1093.

25 O. E. Kalinovskaya and J. K. Vij, The exponential dielectric relaxation dynamics in a secondary alcohol's supercooled liquid and glassy states, J. Chem. Phys., 2000, 112, 3262-3266.

26 D. Fragiadakis, C. M. Roland and R. Casalini, Insights on the origin of the Debye process in monoalcohols from dielectric spectroscopy under extreme pressure conditions, J. Chem. Phys., 2010, 132, 144505.

27 G. Power, M. Nagaraj, J. K. Vij and G. P. Johari, Debye process and dielectric state of an alcohol in a nonpolar solvent, J. Chem. Phys., 2011, 134, 044525.

28 S. P. Bierwirth, J. Bolle, S. Bauer, C. Sternemann, C. Gainaru, M. Tolan and R. Böhmer, in The Scaling of Relaxation Processes, Springer, Cham, 2018, pp. 121-171.

29 S. J. Bass, W. I. Nathan, R. M. Meighan and R. H. Cole, Dielectric Properties of Alkyl Amides. II. Liquid Dielectric Constant and Loss, J. Phys. Chem., 1964, 68, 509-515.

30 R. G. Wassink and P. Bordewijk, Dielectric relaxation of some amides in carbon tetrachloride, Adv. Mol. Relax. Interact. Process., 1978, 13, 299-308.

31 L.-M. Wang and R. Richert, Identification of dielectric and structural relaxations in glass-forming secondary amides, J. Chem. Phys., 2005, 123, 054516.

32 C. Gainaru, S. Bauer, E. Vynokur, H. Wittkamp, W. Hiller, R. Richert and R. Böhmer, Dynamics in Supercooled Secondary Amide Mixtures: Dielectric and Hydrogen Bond Specific Spectroscopies, J. Phys. Chem. B, 2015, 119, 15769-15779.

33 M. Rams-Baron, Z. Wojnarowska, M. Dulski, A. Ratuszna and M. Paluch, Evidence of slow Debyelike relaxation in the anti-inflammatory agent etoricoxib, Phys. Rev. E, 2015, 92, 022309.

34 M. T. Ottou Abe, N. T. Correia, L.-C. Valdes, J. M. B. Ndjaka and F. Affouard, Local molecular organizations of ibuprofen, flurbiprofen and ketoprofen in the liquid phase: Insights from molecular dynamics simulations, J. Mol. Liq., 2015, 205, 74-77.

35 M. T. Ottou Abe, N. T. Correia, J. M. B. Ndjaka and F. Affouard, A comparative study of ibuprofen and ketoprofen glass-forming liquids by molecular dynamics simulations, J. Chem. Phys., 2015, 143, 164506.

36 L.-M. Wang and R. Richert, Debye Type Dielectric Relaxation and the Glass Transition of Alcohols, J. Phys. Chem. B, 2005, 109, 11091-11094.

37 H. Huth, L.-M. Wang, C. Schick and R. Richert, Comparing calorimetric and dielectric polarization modes in viscous 2-ethyl-1-hexanol, J. Chem. Phys., 2007, 126, 104503.

38 C. Gainaru, R. Figuli, T. Hecksher, B. Jakobsen, J. C. Dyre, M. Wilhelm and R. Böhmer, ShearModulus Investigations of Monohydroxy Alcohols: Evidence for a Short-Chain-Polymer Rheological Response, Phys. Rev. Lett., 2014, 112, 098301.

39 A. Kudlik, C. Tschirwitz, S. Benkhof, T. Blochowicz and E. Rössler, Slow secondary relaxation process in supercooled liquids, EPL Europhys. Lett., 1997, 40, 649.

40 G. P. Johari, O. E. Kalinovskaya and J. K. Vij, Effects of induced steric hindrance on the dielectric behavior and $\mathrm{H}$ bonding in the supercooled liquid and vitreous alcohol, J. Chem. Phys., 2001, 114, 4634-4642.

41 R. Böhmer, C. Gainaru and R. Richert, Structure and dynamics of monohydroxy alcoholsMilestones towards their microscopic understanding, 100 years after Debye, Phys. Rep., 2014, 545, 125-195.

$42 \mathrm{~T}$. Hecksher, Communication: Linking the dielectric Debye process in mono-alcohols to density fluctuations, J. Chem. Phys., 2016, 144, 161103.

43 U. Kaatze, R. Behrends and R. Pottel, Hydrogen network fluctuations and dielectric spectrometry of liquids, J. Non-Cryst. Solids, 2002, 305, 19-28.

44 L. P. Singh and R. Richert, Watching hydrogen-bonded structures in an alcohol convert from rings to chains, Phys. Rev. Lett., 2012, 109, 167802.

45 R. Minami, K. Itoh, H. Takahashi and K. Higasi, A theoretical approach to the dielectric relaxation of liquid alcohols, J. Chem. Phys., 1980, 73, 3396-3397. 
46 C. Gainaru, R. Meier, S. Schildmann, C. Lederle, W. Hiller, E. A. Rössler and R. Böhmer, NuclearMagnetic-Resonance Measurements Reveal the Origin of the Debye Process in Monohydroxy Alcohols, Phys. Rev. Lett., 2010, 105, 258303.

47 S. Druot, M. N. Petit, S. Petit, G. Coquerel and N. B. Chanh, Experimental Data and Modelling of the Interactions in Solid State and in Solution between (R) and (S) N-Acetyl- $\alpha$ - Methylbenzylamine. Influence on Resolution by Preferential Crystallization, Mol. Cryst. Liq. Cryst. Sci. Technol. Sect. Mol. Cryst. Liq. Cryst., 1996, 275, 271-291.

$48 \mathrm{E}$. Brown and M. Moudachirou, Agents de dédoublement. 2. Synthèse d'aryluréthanes de l'acide (S)-lactique et leur utilisation dans le dédoublement de bases racémiques, Tetrahedron, 1994, 50, 10309-10320.

49S. Druot Houllemare, Influence des interactions homo- et heterochirales lors du dedoublement par cristallisation preferentielle de deux derives de l'alpha-methylbenzylamine : le n-acetamide et le chloroacetate, Caen, 1999.

50 B. Atawa, N. Couvrat, G. Coquerel, E. Dargent and A. Saiter, Chirality impact on physical ageing: An original case of a small organic molecule, Mater. Lett., 2018, 228, 141-144.

51 G. V. Poel, D. Istrate, A. Magon and V. Mathot, Performance and calibration of the Flash DSC 1, a new, MEMS-based fast scanning calorimeter, J. Therm. Anal. Calorim., 2012, 110, 1533-1546.

52 B. Atawa, N. Couvrat, G. Coquerel, E. Dargent and A. Saiter, Impact of chirality on the Glass Forming Ability and the crystallization from the amorphous state of 5-ethyl-5-methylhydantoin, a chiral poor glass former, Int. J. Pharm., 2018, 540, 11-21.

53 Schaumburg, G. Novocontrol introduces high quality low cost interdigitated comb electrodes. Dielectrics Newsletter 2006, December Issue

54 Z. Chen, A. Sepúlveda, M. D. Ediger and R. Richert, Dielectric spectroscopy of thin films by dualchannel impedance measurements on differential interdigitated electrode arrays, Eur. Phys. J. B, 2012, 85, 268.

55 A. Dhotel, Z. Chen, J. Sun, B. Youssef, J.-M. Saiter, A. Schönhals, L. Tan and L. Delbreilh, From monomers to self-assembled monolayers: the evolution of molecular mobility with structural confinements, Soft Matter, 2014, 11, 719-731.

$56 \mathrm{~S}$. Havriliak and S. Negami, A complex plane representation of dielectric and mechanical relaxation processes in some polymers, Polymer, 1967, 8, 161-210.

57 THE DL POLY 2 USER MANUAL - PDF, http://docplayer.net/14320255-The-dl-poly-2-usermanual.html, (accessed May 31, 2018).

58 J. Wang, R. M. Wolf, J. W. Caldwell, P. A. Kollman and D. A. Case, Development and testing of a general amber force field, J. Comput. Chem., 25, 1157-1174.

59 C. Caleman, P. J. van Maaren, M. Hong, J. S. Hub, L. T. Costa and D. van der Spoel, Force Field Benchmark of Organic Liquids: Density, Enthalpy of Vaporization, Heat Capacities, Surface Tension, Isothermal Compressibility, Volumetric Expansion Coefficient, and Dielectric Constant, J. Chem. Theory Comput., 2012, 8, 61-74.

60 M. Frisch, G. Trucks, H. Schlegel, G. Scuseria, M. Robb, J. Cheeseman, J. Montgomery, T. Vreven, K. Kudin, J. Burant, J. Millam, S. Iyengar, J. Tomasi, V. Barone, B. Mennucci, M. Cossi, G. Scalmani, N. Rega, G. Petersson, H. Nakatsuji, M. Hada, M. Ehara, K. Toyota, R. Fukuda, J. Hasegawa, M. Ishida, T. Nakajima, Y. Honda, O. Kitao, H. Nakai, M. Klene, X. Li, J. Knox, H. Hratchian, J. Cross, V. Bakken, C. Adamo, J. Jaramillo, R. Gomperts, R. Stratmann, O. Yazyev, A. Austin, R. Cammi, C. Pomelli, J. Ochterski, P. Ayala, K. Morokuma, G. Voth, P. Salvador, J. Dannenberg, V. Zakrzewski, S. Dapprich, A. Daniels, M. Strain, O. Farkas, D. Malick, A. Rabuck, K. Raghavachari, J. Foresman, J. Ortiz, Q. Cui, A. Baboul, S. Clifford, J. Cioslowski, B. Stefanov, G. Liu, A. Liashenko, P. Piskorz, I. Komaromi, R. Martin, D. Fox, T. Keith, A. Laham, C. Peng, A. Nanayakkara, M. Challacombe, P. Gill, B. Johnson, W. Chen, M. Wong, C. Gonzalez and J. Pople, Gaussian 03, Revision C.02.

61 D. Wolf, P. Keblinski, S. R. Phillpot and J. Eggebrecht, Exact method for the simulation of Coulombic systems by spherically truncated, pairwise $r-1$ summation, J. Chem. Phys., 1999, 110, 8254-8282. 
62 B. C. Hancock and G. Zografi, Characteristics and Significance of the Amorphous State in Pharmaceutical Systems, J. Pharm. Sci., 1997, 86, 1-12.

63 S. L. Shamblin, L. S. Taylor and G. Zografi, Mixing behavior of colyophilized binary systems, J. Pharm. Sci., 1998, 87, 694-701.

64 M. Naoki, K. Ujita and S. Kashima, Pressure-volume-temperature relations and configurational energy of liquid, crystal, and glasses of D-sorbitol, J. Phys. Chem., 1993, 97, 12356-12362.

65 M. Reading and D. J. Hourston, Eds., Modulated Temperature Differential Scanning Calorimetry: Theoretical and Practical Applications in Polymer Characterisation, Springer Netherlands, 2006.

66 A. Q. Tool and C. G. Eicitlin, Variations Caused in the Heating Curves of Glass by Heat Treatment1, J. Am. Ceram. Soc., 14, 276-308.

67 A. Dhotel, B. Rijal, L. Delbreilh, E. Dargent and A. Saiter, Combining Flash DSC, DSC and broadband dielectric spectroscopy to determine fragility, J. Therm. Anal. Calorim., 2015, 121, 453-461.

68 A. Saiter, C. Devallencourt, J. M. Saiter and J. Grenet, Thermodynamically "strong" and kinetically "fragile" polymeric glass exemplified by melamine formaldehyde resins, Eur. Polym. J., 2001, 37, 1083-1090.

69 A. Saiter, M. Hess, N. A. D'Souza and J. M. Saiter, Entropy and fragility in vitreous polymers, Polymer, 2002, 43, 7497-7504.

70 Y. P. Koh, S. Gao and S. L. Simon, Structural recovery of a single polystyrene thin film using Flash DSC at low aging temperatures, Polymer, 2016, 96, 182-187.

71 C. T. Moynihan, P. B. Macedo, C. J. Montrose, C. J. Montrose, P. K. Gupta, M. A. DeBolt, J. F. Dill, B. E. Dom, P. W. Drake, A. J. Easteal, P. B. Elterman, R. P. Moeller, H. Sasabe and J. A. Wilder, Structural Relaxation in Vitreous Materials*, Ann. N. Y. Acad. Sci., 279, 15-35.

$72 \mathrm{~J}$. E. K. Schawe, Vitrification in a wide cooling rate range: The relations between cooling rate, relaxation time, transition width, and fragility, J. Chem. Phys., 2014, 141, 184905.

73 Y. Z. Chua, G. Schulz, E. Shoifet, H. Huth, R. Zorn, J. W. P. Scmelzer and C. Schick, Glass transition cooperativity from broad band heat capacity spectroscopy, Colloid Polym. Sci., 2014, 292, 18931904.

$74 \mathrm{~J}$. E. K. Schawe, Measurement of the thermal glass transition of polystyrene in a cooling rate range of more than six decades, Thermochim. Acta, 2015, 603, 128-134.

75 A. Hensel, J. Dobbertin, J. E. K. Schawe, A. Boller and C. Schick, Temperature modulated calorimetry and dielectric spectroscopy in the glass transition region of polymers, J. Therm. Anal., 1996, 46, 935-954.

76 A. Hensel and C. Schick, Relation between freezing-in due to linear cooling and the dynamic glass transition temperature by temperature-modulated DSC, J. Non-Cryst. Solids, 1998, 235-237, 510516.

77 S. Weyer, A. Hensel, J. Korus, E. Donth and C. Schick, Broad band heat capacity spectroscopy in the glass-transition region of polystyrene, Thermochim. Acta, 1997, 304-305, 251-255.

78 A. C. Rodrigues, M. T. Viciosa, F. Danède, F. Affouard and N. T. Correia, Molecular Mobility of Amorphous S-Flurbiprofen: A Dielectric Relaxation Spectroscopy Approach, Mol. Pharm., 2014, 11, 112-130.

79 A. Yildirim, P. Szymoniak, K. Sentker, M. Butschies, A. Bühlmeyer, P. Huber, S. Laschat and A. Schönhals, Dynamics and ionic conductivity of ionic liquid crystals forming a hexagonal columnar mesophase, Phys. Chem. Chem. Phys., 2018, 20, 5626-5635.

80 E. Kaminska, M. Tarnacka, O. Madejczyk, A. Chrobok, K. Kaminski and M. Paluch, Observation of the nearly constant loss in super rigid saccharides: in search of a hidden crossover in dynamics deep in the glassy state, Phys. Chem. Chem. Phys., 2016, 18, 8901-8910.

$81 \mathrm{M}$. Wübbenhorst and J. van Turnhout, Analysis of complex dielectric spectra. I. One-dimensional derivative techniques and three-dimensional modelling, J. Non-Cryst. Solids, 2002, 305, 40-49.

82 Q. Viel, L. Delbreilh, G. Coquerel, S. Petit and E. Dargent, Molecular Mobility of an Amorphous Chiral Pharmaceutical Compound: Impact of Chirality and Chemical Purity, J. Phys. Chem. B, 2017, 121, 7729-7740. 
83 K. L. Ngai, An extended coupling model description of the evolution of dynamics with time in supercooled liquids and ionic conductors, J. Phys. Condens. Matter, 2003, 15, S1107.

84 K. Adrjanowicz, Z. Wojnarowska, P. Wlodarczyk, K. Kaminski, M. Paluch and J. Mazgalski, Molecular mobility in liquid and glassy states of Telmisartan (TEL) studied by Broadband Dielectric Spectroscopy, Eur. J. Pharm. Sci., 2009, 38, 395-404.

85 M. Paluch, C. M. Roland, S. Pawlus, J. Zioło and K. L. Ngai, Does the Arrhenius Temperature Dependence of the Johari-Goldstein Relaxation Persist above $\$\{T\}\{g\} \$$ ?, Phys. Rev. Lett., 2003, 91, 115701.

86 K. L. Ngai and M. Paluch, Classification of secondary relaxation in glass-formers based on dynamic properties, J. Chem. Phys., 2003, 120, 857-873.

87 F. Alvarez, A. Alegría and J. Colmenero, Interconnection between frequency-domain HavriliakNegami and time-domain Kohlrausch-Williams-Watts relaxation functions, Phys. Rev. B, 1993, 47, 125-130.

88 K. L. Ngai and S. Capaccioli, Relation between the activation energy of the Johari-Goldstein lensuremath $\{\backslash$ beta\} relaxation and $\$\{T\}\{g\} \$$ of glass formers, Phys. Rev. E, 2004, 69, 031501.

89 H. VOGEL, Das Temperaturabhangigkeitsgesetz der Viskositat von Flussigkeiten, Phys Z, 1921, 22, 645-646.

90 G. S. Fulcher, Analysis of Recent Measurements of the Viscosity of Glasses, J. Am. Ceram. Soc., 1925, 8, 339-355.

91 G. Tammann and W. Hesse, Die Abhängigkeit der Viscosität von der Temperatur bie unterkühlten Flüssigkeiten, Z. Für Anorg. Allg. Chem., 1926, 156, 245-257.

92 R. Böhmer, K. L. Ngai, C. A. Angell and D. J. Plazek, Nonexponential relaxations in strong and fragile glass formers, J. Chem. Phys., 1993, 99, 4201-4209.

93 L.-M. Wang and R. Richert, Dynamics of glass-forming liquids. IX. Structural versus dielectric relaxation in monohydroxy alcohols, J. Chem. Phys., 2004, 121, 11170-11176.

94 A. Mandanici, W. Huang, M. Cutroni and R. Richert, Dynamics of glass-forming liquids. XII. Dielectric study of primary and secondary relaxations in ethylcyclohexane, J. Chem. Phys., 2008, 128, 124505.

95 C. J. F. Böttcher, in Theory of Electric Polarization (Second Edition), Elsevier, Amsterdam, 1973, pp. 9-58.

$96 \mathrm{~S}$. S. N. Murthy and M. Tyagi, Experimental study of the high frequency relaxation process in monohydroxy alcohols, J. Chem. Phys., 2002, 117, 3837-3847.

97 S. Pal, B. Bagchi and S. Balasubramanian, Hydration Layer of a Cationic Micelle, C10TAB: Structure, Rigidity, Slow Reorientation, Hydrogen Bond Lifetime, and Solvation Dynamics, J. Phys. Chem. B, 2005, 109, 12879-12890.

98 P. Derollez, A. Hédoux, Y. Guinet, F. Danède and L. Paccou, Structure determination of the crystalline phase of $\mathrm{n}$-butanol by powder $\mathrm{X}$-ray diffraction and study of intermolecular associations by Raman spectroscopy, Acta Crystallogr. Sect. B Struct. Sci. Cryst. Eng. Mater., 2013, 69, 195-202.

99 F. Affouard and N. T. Correia, Debye Process in Ibuprofen Glass-Forming Liquid: Insights from Molecular Dynamics Simulation, J. Phys. Chem. B, 2010, 114, 11397-11402.

100 A. Lerbret, P. Bordat, F. Affouard, M. Descamps and F. Migliardo, How Homogeneous Are the Trehalose, Maltose, and Sucrose Water Solutions? An Insight from Molecular Dynamics Simulations, J. Phys. Chem. B, 2005, 109, 11046-11057. 